\title{
Exploring the Universe with Metal-Poor Stars
}

\author{
Anna Frebel
}

\begin{abstract}
The early chemical evolution of the Galaxy and the Universe is vital to our understanding of a host of astrophysical phenomena. Since the most metal-poor Galactic stars (with metallicities down to $[\mathrm{Fe} / \mathrm{H}] \sim-5.5$ ) are relics from the highredshift Universe, they probe the chemical and dynamical conditions of the Milky Way and the origin and evolution of the elements through nucleosynthesis. They also provide constraints on the nature of the first stars, their associated supernovae and initial mass function, and early star and galaxy formation. The Milky Way's dwarf satellites contain a large fraction $(\sim 30 \%)$ of the known most metal-poor stars that have chemical abundances that closely resemble those of equivalent halo stars. This suggests that chemical evolution may be universal, at least at early times, and that it is driven by massive, energetic SNe. Some of these surviving, ultra-faint systems may show the signature of just one such Pop III star; they may even be surviving first galaxies. Early analogs of the surviving dwarfs may thus have played an important role in the assembly of the old Galactic halo whose formation can now be studied with stellar chemistry. Following the cosmic evolution of small halos in simulations of structure formation enables tracing the cosmological origin of the most metalpoor stars in the halo and dwarf galaxies. Together with future observations and additional modeling, many of these issues, including the reionization history of the Milky Way, may be constrained this way. The chapter concludes with an outlook about upcoming observational challenges and ways forward is to use metal-poor stars to constrain theoretical studies.
\end{abstract}

Anna Frebel

Harvard-Smithsonian Center for Astrophysics, 60 Garden St., Cambridge, MA 02138, USA,

e-mail: afrebelecfa.harvard.edu 


\section{Introduction}

As Carl Sagan once remarked, If you wish to make an apple pie from scratch, you must first create the Universe. An apple contains at least 16 different elements, and the human body is even more complex, having at least trace amounts of nearly 30 element 1 , all owing to a 14-billion year long manufacturing process called cosmic chemical evolution. Thus, the basis of chemically complex and challenging undertakings such as cooking and baking, not to mention the nature of life, will ultimately be gained through an understanding of the formation of the elements that comprise organic material. It is thus important to examine how the constituents of an apple, and by extension the stuff of life and the visible Universe were created: baryonic matter in the form of elements heavier than primordial hydrogen and helium.

This chapter aims at describing that the chemical abundances observed in the most metal-poor stars can be employed to unravel a variety of details about the young Universe, such as early star formation environments, supernovae ( $\mathrm{SNe}$ ) nucleosynthesis, and the formation process(es) of the Galactic halo. To illustrate the meaning of low-metallicity, Figure 1 shows the progression from metal-rich to the most metal-poor stars; spectra around the strongest optical Fe line at $3860 \AA$ are shown of the Sun and three metal-poor main-sequence turn-off stars. The number of atomic absorption lines detectable in the spectra decreases with increasing metaldeficiency. In HE 1327-2326, the star with the currently low Fe abundance, only the intrinsically strongest metal lines remain observable. As can be seen in the Figure, these are extremely weak. If a main-sequence star with even lower Fe value (or somewhat hotter temperature) was discovered, no Fe lines would be measurable anymore. In the case of a metal-deficient giant, the lines would be somewhat stronger due to its cooler temperature and thus allow for the discovery of an object with $[\mathrm{Fe} / \mathrm{H}] \lesssim-6$.

Because these most metal-poor stars represent easily accessible local equivalents of the high-redshift universe, and as such, provide a unique tool to address a wide range of near and far-field cosmological topics. In short, metal-poor stars enable scientific progress in three areas that bridge our understanding of the current state of the Galactic halo and its old stellar population with that of the evolution of local dwarf galaxies, to the formation of large galaxies like the Milky Way more generally, as well as the beginning of star and galaxy formation in the early universe.

1. Stellar Archaeology: Constrains the astrophysical sites and conditions of nucleosynthesis and the major physical processes that drove early star formation. Abundance measurements of many elements throughout the periodic table of metal-poor halo stars enable the detailed documentation of the earliest chemical enrichment events.

2. Dwarf Archaeology: Provides constraints on galaxy formation on small scales, and metal mixing and feedback processes. By comparing abundances of metalpoor stars in ultra-faint dwarf galaxies to those of equivalent halo stars, the universality of the (beginning of) chemical evolution can be tested, what the relation

\footnotetext{
${ }^{1}$ http://chemistry.about.com/cs/howthingswork/f/blbodyelements.htm
} 
is between the dwarfs and the "building blocks" of the Galactic halo, and whether they are the survivors of the first galaxies.

3. Near-Field Cosmology: Determines the role of metal-poor stars as tracers of the accretion history of the Milky Way halo. The coupling of low-metallicity stellar abundances with results from cosmological simulations enables the study of the formation mechanism(s) of large galaxies like the Milky Way with its old halo and satellites.

For stellar archaeology, large numbers of Galactic metal-poor halo stars, mostly found in objective-prism surveys in both hemispheres such as the HK survey of Beers and collaborators, the Hamburg/ESO survey (Christlieb and collaborators), and more recently SDSS, are needed to gain detailed insights into the history and evolution of our Galaxy (e.g., Beers \& Christlieb 2005; Frebel \& Norris 2011). For dwarf archaeology, observations of any stars in dwarf satellite galaxies orbiting the Milky Way are required, although these are more difficult to obtain than those of the halo stars. Finally, near-field cosmology encourages the systematic use of metalpoor stars for studying galaxy formation and cosmological aspects. In a universe dominated by cold dark matter (CDM), like the one we live in, galaxy formation proceeds hierarchically through the accretion of smaller objects onto the main halo. Simulations show that successive growth is reflected in the abundance of dark matter substructures in the halos of large galaxies like the Milky Way, and it is believed that the luminous satellites of our Galaxy are the visible counterparts to at least some of these substructures. Thus, the collective body of metal-poor stars now found in the halo as well as the dwarf galaxies enable addressing a number of important, outstanding questions that show how closely connected the three topics are.

- What is the nature of Pop III stars? Are the yields of the first SNe different from today's? Can the signatures of theorized pair-instability $\mathrm{SNe}$ be found in metalpoor stars?

- What drove early star formation? How/where did the first low-mass stars and the first galaxies form?

- What are the main nucleosynthesis processes and sites that are responsible for forming the elements from the Big Bang until today?

- How did chemical evolution proceed? How do stellar chemistry and halo kinematics correlate? How can the abundances be used to learn about the halo formation process?

- Was the old halo built from accreted satellites? Can accreted dwarf galaxies be identified in the halo? Did the first stars form in dwarf galaxies?

Each section of this chapter discusses a significant aspect in which metal-poor stars offer unique insight into the young universe. Section 2 sets the overall stage by introducing the first stars, the halo metallicity distribution function and considerations regarding early low-mass star formation. Section 3 describes the role of metalpoor stars in the Galactic as tracers of the earliest enrichment events and chemical evolution. This concept is extended to dwarf galaxies in Section 4. Understanding the formation history of the Milky Way with the help of metal-poor stars is outlined in Section 5. Conclusions and an outlook at given in Section 6. 


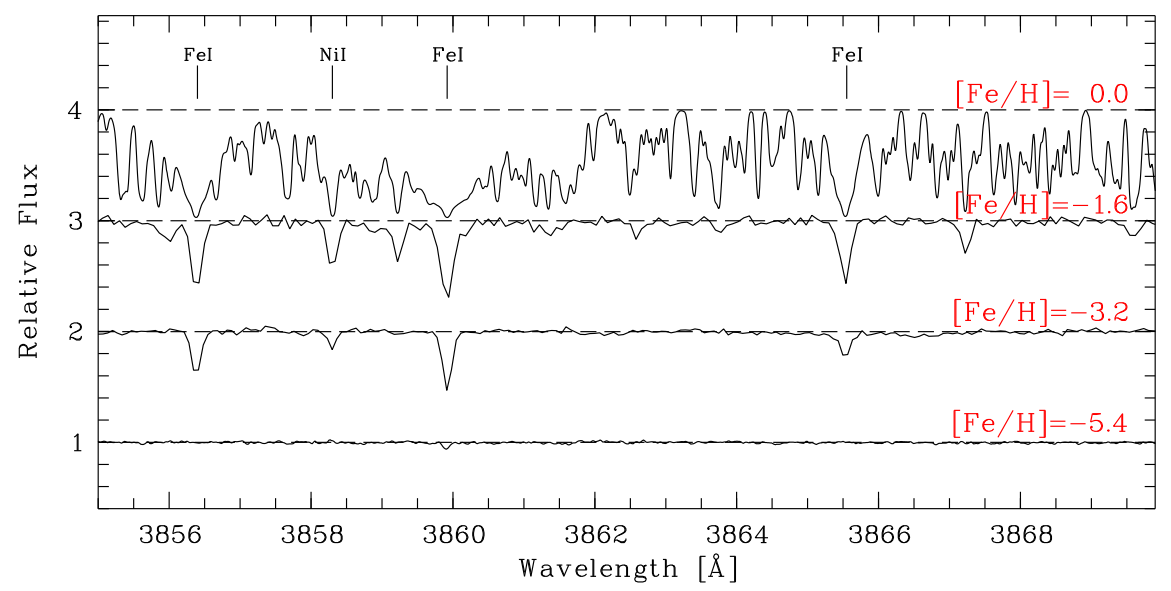

Fig. 1 Spectral comparison of stars in the main-sequence turn-off region with different metallicities. Several absorption lines are marked. The variations in line strength reflect the different metallicities. From top to bottom: Sun with $[\mathrm{Fe} / \mathrm{H}]=0.0, \mathrm{G} 66-30[\mathrm{Fe} / \mathrm{H}]=-1.6$ (Norris et al. 1997) $\mathrm{G} 64-12[\mathrm{Fe} / \mathrm{H}]=-3.2$ (Aoki et al. 2006), and HE1327-2326 $[\mathrm{Fe} / \mathrm{H}]=-5.4$ (Frebel et al. 2005). Figure taken from Frebel (2010).

\section{Further Reading \& Definitions}

This chapter is partially based on an article by Frebel (2010) 2 that, among other topics, introduces metal-poor stars as probes for theoretical works related to the early universe. Here, this discussion is extended to showcase the versatility and potential of the oldest stars for dwarf archaeology and near-field cosmology. The main aim is to outline the broad picture of studying galaxy formation processes with stellar chemistry. For a more in-depth discussion of stellar abundances, abundance derivations, details on nucleosynthesis and chemical evolution, kinematics, stellar age determinations and cosmogony, the reader is referred to Frebel \& Norris (2011) and references therein.

Since there exist a large range of metal-poor stars in terms of their metallicities and chemical signatures, Beers \& Christlieb (2005) suggested a classification scheme. Extensive use will be made of their term "extremely metal-poor stars", referring to stars with $[\mathrm{Fe} / \mathrm{H}]<-3.0$. This nomenclature shows that the main metallicity indicator used to determine any stellar metallicity is the iron abundance, $[\mathrm{Fe} / \mathrm{H}]$, which is defined as $[\mathrm{A} / \mathrm{B}]=\log _{10}\left(N_{\mathrm{A}} / N_{\mathrm{B}}\right)_{\star}-\log _{10}\left(N_{\mathrm{A}} / N_{\mathrm{B}}\right)_{\odot}$ for the number $\mathrm{N}$ of atoms of elements $\mathrm{A}$ and $\mathrm{B}$, and $\odot$ refers to the Sun. For example, $[\mathrm{Fe} / \mathrm{H}]=-3.0$ is $1 / 1000$ of solar $\mathrm{Fe}$ abundance. With few exceptions, $[\mathrm{Fe} / \mathrm{H}]$ traces the overall metallicity of the objects fairly well.

2 The 2009 Biermann Award Lecture, originally published in Astronomische Nachrichten by VCH/Wiley, 2010, 331, 474 


\section{Exploring The Early Universe with Metal-Poor Stars}

\subsection{The First Stars}

According to cosmological simulations that are based on the $\Lambda$ cold dark matter model of hierarchical structure growth in the Universe, the first stars formed in small minihalos some few hundred million years after the Big Bang. Due to the lack of cooling agents in the primordial gas, significant fragmentation was largely suppressed so that these first objects were very massive (of the order to $\sim 100 \mathrm{M}_{\odot}$; e.g, Bromm \& Yoshida (2011) and references therein). This is in contrast to lowmass stars dominating today's mass function. These objects are referred to as Population III (Pop III) as they formed from metal-free gas. Recent modeling of first star formation suggests that these early behemoth were rapidly rotating (Stacy et al. 2010) and new observations have provided evidence in support of this claim (Chiappini et al. 2011). Moreover, significant fragmentation of the star forming cloud may occur that could lead to multiple first stars in a given minihalo (Clark et al. 2011).

The stars soon exploded as SNe to either collapse into black holes (progenitor masses of $25<M_{\odot}<140$ and $M_{\odot}>260$ ) or to die as energetic pair-instability SNe (PISN; $140<M_{\odot}<260$; Heger et al. 2002). During their deaths, these objects provided vast amounts of ionizing radiation (and some of the first metals in the case of the PISNe) that changed the conditions of the surrounding material for subsequent star formation even in neighboring minihalos. Hence, the second generation of stars might have been less massive $\left(\mathrm{M}_{\star} \sim 10 \mathrm{M}_{\odot}\right)$. Partially ionized gas supports the formation of the $\mathrm{H}_{2}$, and then the HD molecule, which in turn facilitates more effective cooling than what is possible in neutral gas. Also, any metals or dust grains left behind from PISNe would have similar cooling effects. This may then have led to the first more regular metal-producing SNe, although not all higher mass SNe must necessarily end in black hole formation. Umeda \& Nomoto (2003) suggested that some $25 \mathrm{M}_{\odot}$ stars undergo only a partial fallback, so that only some of the newly created metals get ejected into the surrounding gas.

By that time, most likely enough metals were present to ensure sufficient gas fragmentation to allow for low-mass $\left(<1 \mathrm{M}_{\odot}\right)$ star formation. Stars that formed from any metal-enriched material are referred to as Population II (Pop II) stars. More metal-rich stars like the Sun that formed in a much more metal-rich Universe are called Population I. Studying the "chemical fingerprints" of the oldest, most metalpoor stars (extreme Pop II) reveals information about the first nucleosynthesis events in the Universe; indeed, several metal-poor star abundance patterns have been fitted with calculated Pop III SN yields (see Section 3.3. Moreover, evidence for the existence of PISNe could potentially be obtained if their characteristic signature (a pronounced effect in the abundance signature of elements with odd or even atomic number) were found in metal-poor stars. This has, however, not yet occurred. 


\subsection{The Metallicity Distribution Function of the Galactic Halo}

The metallicity distribution function (MDF) represents the integrated chemical evolution of a system which began with the first stars and was continued by many stellar generations at various astrophysical sites and over different timescales.

To establish the MDF of a given system, a large, complete sample of stars with good metallicity estimates is required. Over the past two decades, the quest to find the most metal-poor stars to study the chemical evolution of the Galaxy led to a significant number of stars with metallicities down to $[\mathrm{Fe} / \mathrm{H}] \sim-4.0$ (see Beers \& Christlieb 2005 for a more detailed review). Those stars were initially selected as candidates from a large survey, such as the HK survey (Beers et al. 1992) and the Hamburg/ESO survey (Wisotzki et al. 1996). A large survey is required to provide numerous low-resolution spectra to search for weak-lined stellar candidates indicating metal deficiency. Those spectra have to cover the strong Ca II K line at $3933 \AA$ because the strength of this line indicates the metallicity of the star, and can be measured even in low-quality spectra. This is shown in Figure 2 If this line is sufficiently weak as a function of the star's estimated effective temperature, an object is selected as a candidate metal-poor star. For all candidates, medium-resolution spectra $(R \sim 2,000)$ are required to more accurately determine the Ca II K line strength for a more robust estimate for the Fe abundance. This line is still the best indicator for the overall metallicity $[\mathrm{Fe} / \mathrm{H}]$ of a metal-poor star in such spectra. In the Sloan Digital Sky Survey and LAMOST survey, the survey spectra themselves are already of medium-resolution, allowing for a quicker and more direct search for metal-poor stars. Photometric surveys like Skymapper with extensive filter sets designed for stellar work (Keller et al. 2007) will also yield large numbers of high-quality candidates.

To confirm the metallicity, and to measure elemental abundances from their respective absorption lines besides that of iron, high-resolution $(R>20,000)$ optical spectroscopy is required (see bottom panel of Figure 2). Only then the various elements become accessible for studying the chemical evolution of the Galaxy. Those elements include carbon, magnesium, calcium, titanium, nickel, strontium, and barium, and trace different enrichment mechanisms, events and timescales. Abundance ratios $[\mathrm{X} / \mathrm{Fe}]$ as a function of $[\mathrm{Fe} / \mathrm{H}]$ can then be derived for the lighter elements $(Z<30)$ and neutron-capture elements $(Z>38)$. The resulting abundance trends will be further described in Section 3.2. The final number of elements thereby depends on the type of metal-poor star, the wavelength coverage of the data, and the data quality itself.

Schörck et al. (2009) and Li et al. (2010) presented MDFs for halo stars that are corrected for various selection effects and other biases. The number of known metalpoor stars declines significantly with decreasing metallicity (below $[\mathrm{Fe} / \mathrm{H}]<-2.0$ ) as illustrated in Figure 3 . Only very few stars are known $(\lesssim 30)$ with metallicities below $[\mathrm{Fe} / \mathrm{H}]<-3.5$, but it is these objects that enable the most insight into the early universe and the beginning of chemical evolution.

The bias-corrected MDF shows how rare metal-poor stars really are, but also, that past targeted ("biased") searches for metal-poor stars have been extremely success- 


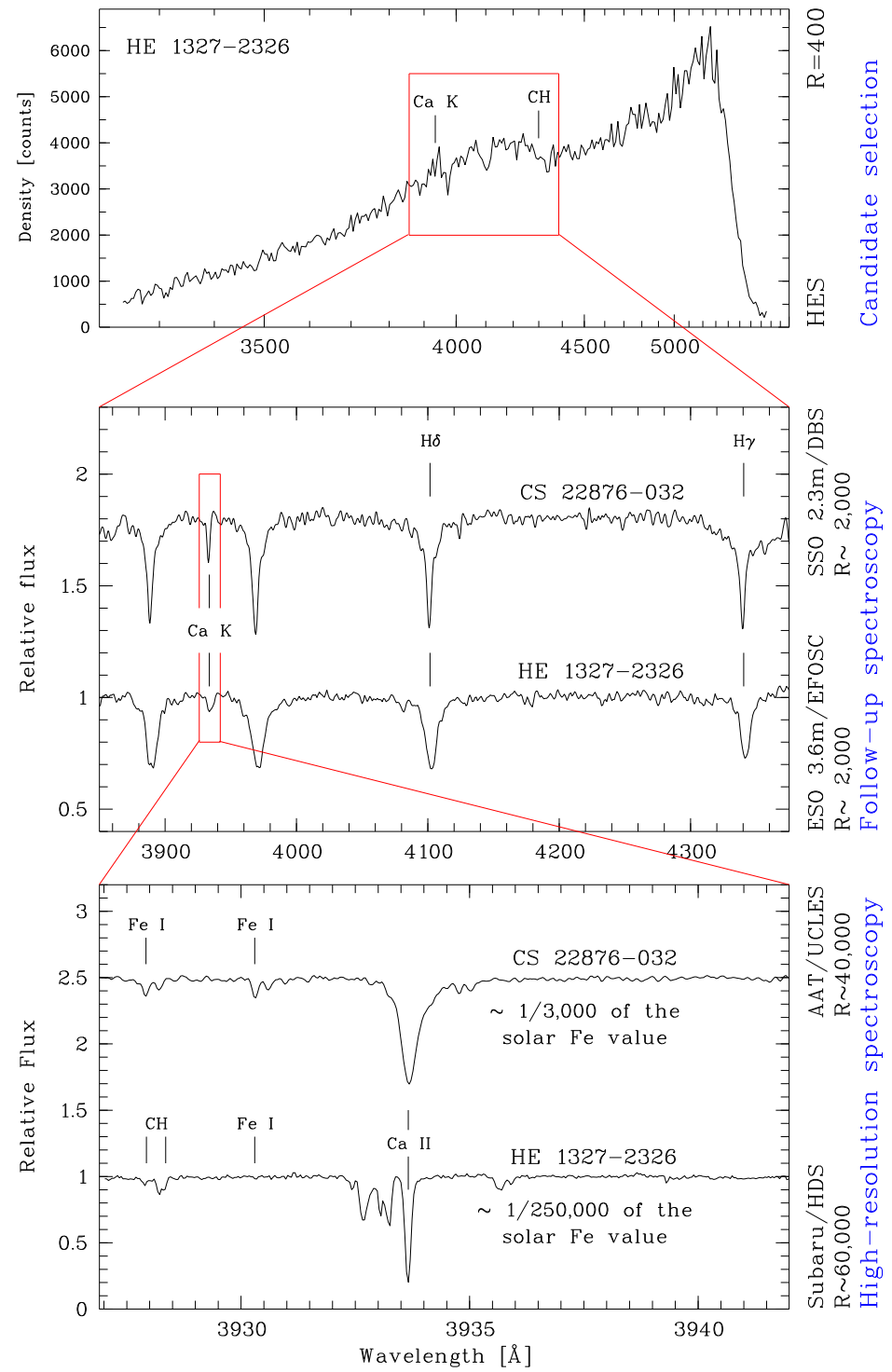

Fig. 2 The three observational steps to find metal-poor stars illustrated by means of HE 1327-2326. Top panel: HES objective-prism spectrum. Middle panel: Medium-resolution spectrum of HE $1327-2326$ in comparison with CS $22876-032([\mathrm{Fe} / \mathrm{H}]=-3.7$; Norris et al. 2000 and references therein). From this data we measured $[\mathrm{Fe} / \mathrm{H}]=-4.3$ for $\mathrm{HE} 1327-2326$ because interstellar Ca blended with the Ca II K line. Bottom panel: High-resolution spectra of both objects. Only with the high-resolution data was it possible to determine the true iron abundance, $[\mathrm{Fe} / \mathrm{H}]=-5.4$, for HE 1327-2326. Figure taken from Frebel et al. (2005b). 
ful at identifying these rare objects (e.g., Frebel et al. 2006; Christlieb et al. 2008). The most important achievements in terms of the most iron-deficient stars was the push to a significantly lower stellar metallicity $[\mathrm{Fe} / \mathrm{H}]$ almost a decade ago: From a longstanding $[\mathrm{Fe} / \mathrm{H}]=-4.0\left(\mathrm{CD}-38^{\circ} 245\right.$; Bessell \& Norris 1984$)$ to $[\mathrm{Fe} / \mathrm{H}]=$ -5.2 (HE 0107-5240; Chrislieb et al. 2002 3 ), and down to $[\mathrm{Fe} / \mathrm{H}]=-5.4$ more recently (HE 1327-2326; Frebel et al. 2005). Overall, only three stars are known with iron abundances of $[\mathrm{Fe} / \mathrm{H}]<-4.0$. The third star, HE $0557-4840$ Norris et al. 2007 with $[\mathrm{Fe} / \mathrm{H}]<-4.8$, bridges the gap between $[\mathrm{Fe} / \mathrm{H}]=-4.0$ and the two hyper Fe-poor objects.

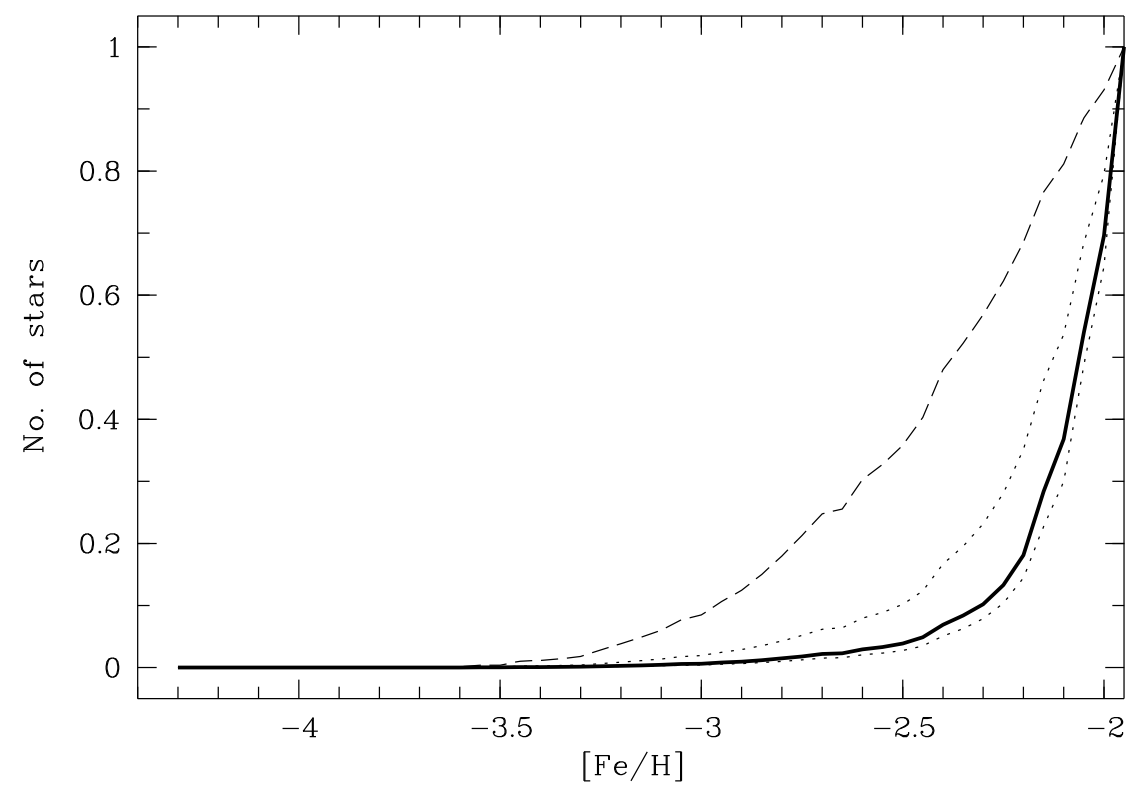

Fig. 3 Cumulative metallicity distribution function of the Galactic halo based on metal-poor giants selected form the Hamburg/ESO survey (Schöerck et al. 2009). The solid line represents the biascorrected MDF, whereas the dotted lines show the level of uncertainty in the MDF based on the various correction functions. The dashed line shows the uncorrected, "as observed" MDF - The difference to the solid line shows how successful searches for the most metal-poor stars have been.

Beyond these individual stars that form the very tail of the MDF, a number of studies involving samples of $\sim 10-30$ stars in the metallicity range of $-4.0[\mathrm{Fe} / \mathrm{H}]<$ -2.5 have been carried out over the years. These works have delivered many important details about chemical enrichment and nucleosynthesis, and greatly improved our understanding of the early universe (e.g., McWilliam et al. 1995, Ryan et al.

3 Applying the same non-LTE correction to the FeI abundances of HE 0107-5240 and HE $1327-2326$ leads to a final abundance of $[\mathrm{Fe} / \mathrm{H}]=-5.2$ for $\mathrm{HE} 0107-5240$. 
1996, Cayrel et al. 2004, Barklem et al. 2005, Aoki et al. 2008, Cohen et al. 2008, Lai et al. 2008, Hollek et al. 2011).

\subsection{Early low-mass star formation and the connection to carbon-enhanced metal-poor stars}

Early Pop II stars began to form from the enriched material left behind by the first stars. The actual formation process(es) of these initial low-mass $\left(\mathrm{M} \leq 0.8 \mathrm{M}_{\odot}\right)$ Pop II stars (i.e. the most metal-poor stars) that live longer than a Hubble time, are, however, not well understood so far. Ideas for the required cooling processes necessary to induce sufficient fragmentation of the near-primordial gas include cooling through metal enrichment ("critical metallicity") or dust, cooling based on enhanced molecule formation due to ionization of the gas, as well as more complex effects such as turbulence and magnetic fields (Bromm et al. 2009).

Fine-structure line cooling through neutral carbon and singly-ionized oxygen has been suggested as a main cooling agent facilitating low-mass star formation (Bromm $\&$ Loeb 2003). These elements were likely produced in vast quantities in Pop III objects (e.g. Meynet et al. 2006; Chiappini et al. 2011). Gas fragmentation is then induced once a critical metallicity of the interstellar medium (ISM) is achieved. The existence of such a critical metallicity can be probed with large numbers of carbon and oxygen-poor metal-poor stars. Frebel et al. (2007b) developed an "observerfriendly" description of the critical metallicity that incorporates the observed $\mathrm{C}$ and/or O stellar abundances; $D_{\text {trans }}=\log \left(10^{[\mathrm{C} / \mathrm{H}]}+0.3 \times 10^{[\mathrm{O} / \mathrm{H}]}\right) \geq-3.5$. Any lowmass stars still observable today then has to have $\mathrm{C}$ and/or $\mathrm{O}$ abundances above the threshold of $D_{\text {trans }}=-3.5$ (see Figure 1 in Frebel et al. 2007). At metallicities of $[\mathrm{Fe} / \mathrm{H}] \gtrsim-3.5$, most stars have $\mathrm{C}$ and/or $\mathrm{O}$ abundances that are above the threshold since they follow the solar $\mathrm{C}$ and $\mathrm{O}$ abundances simply scaled down to their respective $\mathrm{Fe}$ values. Naturally, this metallicity range is not suitable for directly probing the first low-mass stars. Below $[\mathrm{Fe} / \mathrm{H}] \sim-3.5$, however, the observed $\mathrm{C}$ and/or $\mathrm{O}$ levels must be higher than the Fe-scaled solar abundances to be above the critical metallicity. Indeed, none of the known lowest-metallicity stars has a $D_{\text {trans }}$ below the critical value, consistent with this cooling theory. Some stars, however, have values very close to $D_{\text {trans }}=-3.5$. $\mathrm{HE} 0557-4840$, at $[\mathrm{Fe} / \mathrm{H}]=-4.75$ (Noris et al. 2007), falls just onto the critical limit (M. Bessell 2009, priv. comm.). A star in the ultrafaint dwarf galaxy Boötes I has $D_{\text {trans }}=-3.2($ at $[\mathrm{Fe} / \mathrm{H}]=-3.7$; and assuming that its oxygen abundance is twice that of carbon). Another interesting case is the most metal-poor star in the classical dwarf galaxy Sculptor, which has an upper limit of carbon of $[\mathrm{C} / \mathrm{H}]<-3.6$ at $[\mathrm{Fe} / \mathrm{H}]=-3.8$ (Frebel et al. 2010b). Despite some still required up-correction of carbon to account for atmospheric carbon-depletion of this cool giant, the star could potentially posses a sub-critical $D_{\text {trans }}$ value.

Overall, more such "borderline" examples are crucial to test for the existence of a critical metallicity. If fine-structure line cooling were the dominant process for low-mass star formation, two important consequences would follow: 1) Future stars 
to be found with $[\mathrm{Fe} / \mathrm{H}] \lesssim-4.0$ are predicted to have these substantial $\mathrm{C}$ and/or $\mathrm{O}$ overabundances with respect to Fe. 2) The so-far unexplained large fraction of metal-poor objects that have large overabundances of carbon with respect to iron $([\mathrm{C} / \mathrm{Fe}]>1.0)$ may reflect an important physical cause. About $20 \%$ of metal-poor stars with $\mathrm{Fe} / \mathrm{H} \lesssim-2.5$ exhibit this behavior (e.g. Beers \& Christlieb 2005). Moreover, at the lowest metallicities, this fraction is even higher. All three stars with $[\mathrm{Fe} / \mathrm{H}]<-4.0$ are extremely $\mathrm{C}$-rich, well in line with the prediction of the line cooling theory.

This may, however, not the only way for forming low-mass stars. Cooling through dust grains might also have been responsible for the transition from Pop III to Pop II star formation. Dust created in high-density regions during the first $\mathrm{SNe}$ explosions or mass loss during the evolution of Pop III stars may induce fragmentation processes (e.g., Schneider et al. 2006) that lead to the formation of subsolar-mass stars. The critical metallicity in this scenario is a few orders of magnitude below that of $\mathrm{C}$ and $\mathrm{O}$ line cooling. If some metal-poor stars are found to be significantly below $D_{\text {trans }}=-3.5$, their existence may still be consistent with the critical value set by dust cooling. Irrespective of the differences in cooling channels, such criteria will need to be incorporated in large-scale simulations to take environmental influences, such as the available gas mass, into account.

\section{Stellar Archaeology}

\subsection{Validating Stellar Archaeology}

The concept of stellar archaeology is based on long-lived low-mass metal-poor main-sequence and giant stars whose chemical abundances are thought to reflect the composition of the gas cloud during their formation period. A vital assumption is that the stellar surface compositions have not been significantly altered by any internal mixing processes given that these stars are fairly unevolved despite their old age. But are there other means by which the surface composition could be modified? Accretion of interstellar matter while a star orbits in the Galaxy for $\sim 10 \mathrm{Gyr}$ has long been suggested as a mechanism to affect the observed abundance patterns. Iben (1983) calculated a basic "pollution limit" of $[\mathrm{Fe} / \mathrm{H}]=-5.7$ based on Bondi-Hoyle accretion. He predicted that no stars with Fe abundances below this value could be identified as such since they would have accreted too much enriched material.

Assuming that stars with such low-metallicities exist (for example low-mass Pop III stars if the IMF was Salpeter-like, and not top-heavy), significant amounts of interstellar accretion could masquerade the primordial abundances of those putative low-mass Pop III stars. Analogously, stars with very low abundances, say $[\mathrm{Fe} / \mathrm{H}]<-5.0$, could principally be affected also. To assess the potential accretion level, Frebel et al. (2009) carried out a kinematic analysis of a sample of metal-poor stars to assess their potential accretion histories over the past $10 \mathrm{Gyr}$ in a Milky 
Way-like potential. The amount of accreted Fe was calculated based on the total accreted material over $10 \mathrm{Gyr}$. The overall chemical evolution with time was taken into account assuming the ISM to have scaled solar abundances. The stellar abundances were found to be little affected by accretion given their generally fast space velocities. The calculated, "accreted abundances" were often lower than the observed measurements by several orders of magnitude. Johnson et al. (2011), on the other hand, investigated direct accretion onto primordial low-mass stars. If these stars had a weak solar-like wind it would prevent the accretion of any material, at least in the early universe. This would also be true for any low-metallicity stars, although it was not considered by Frebel et al. (2009).

Generally, these studies show that accretion does not significantly alter the observed abundance patterns, even in an extreme case in which a star moves once through a very large, dense cloud. The concept of stellar archaeology can thus be deemed viable. Nevertheless, since there is a large accretion dependency on the space velocity it becomes obvious that kinematic information is vital for the identification of the lowest-metallicity stars in the Milky Way and the interpretation of their abundances. A way forward would be an extensive assessment of potential gas accretion histories for stars throughout the hierarchical assembly of a large galaxy. However, the uncertainties regarding the existence and extent of stellar winds may prevent strong conclusions.

\subsection{Chemical Evolution of the Galaxy}

Generally, there are several main groups of elements observed in metal-poor stars, with each group having a common, main production mechanism; 1) $\alpha$-elements (e.g. $\mathrm{Mg}, \mathrm{Ca}, \mathrm{Ti}$ ) are produced through $\alpha$-capture during various burning stages of late stellar evolution, before and during SN explosions. These yields appear very robust with respect to parameters such as mass and explosion energy 2) Fe-peak elements $(23<Z<30)$ are synthesized in a host of different nucleosynthesis processes before and during SN explosions such as radioactive decay of heavier nuclei or direct synthesis in explosive burning stages, neutron-capture onto lower-mass Fe-peak elements during helium and later burning stages and $\alpha$-rich freeze-out processes. Their yields also depend on the explosion energy; 3) Light and heavy neutroncapture elements $(Z>38)$ are either produced in the slow (s-) process occurring in thermally pulsing AGB stars (and then transferred to binary companions or deposited into the ISM through stellar winds) or in the rapid (r-) process most likely occurring in core-collapse SN explosions. For more details on SN nucleosynthesis see e.g., Woosley \& Weaver (1995).

The $\alpha$-element abundances in metal-poor halo stars with $[\mathrm{Fe} / \mathrm{H}]<-1.5$ are enhanced by $\sim 0.4$ dex with respect to Fe as seen in Figure 4. This reflects a typical core-collapse SN signature because at later times (in chemical space at about $[\mathrm{Fe} / \mathrm{H}] \sim-1.5)$ the onset of SN Ia provides a significant contribution to the overall Galactic Fe inventory. As a consequence, the $[\alpha / \mathrm{Fe}]$ ratio decreases down to 

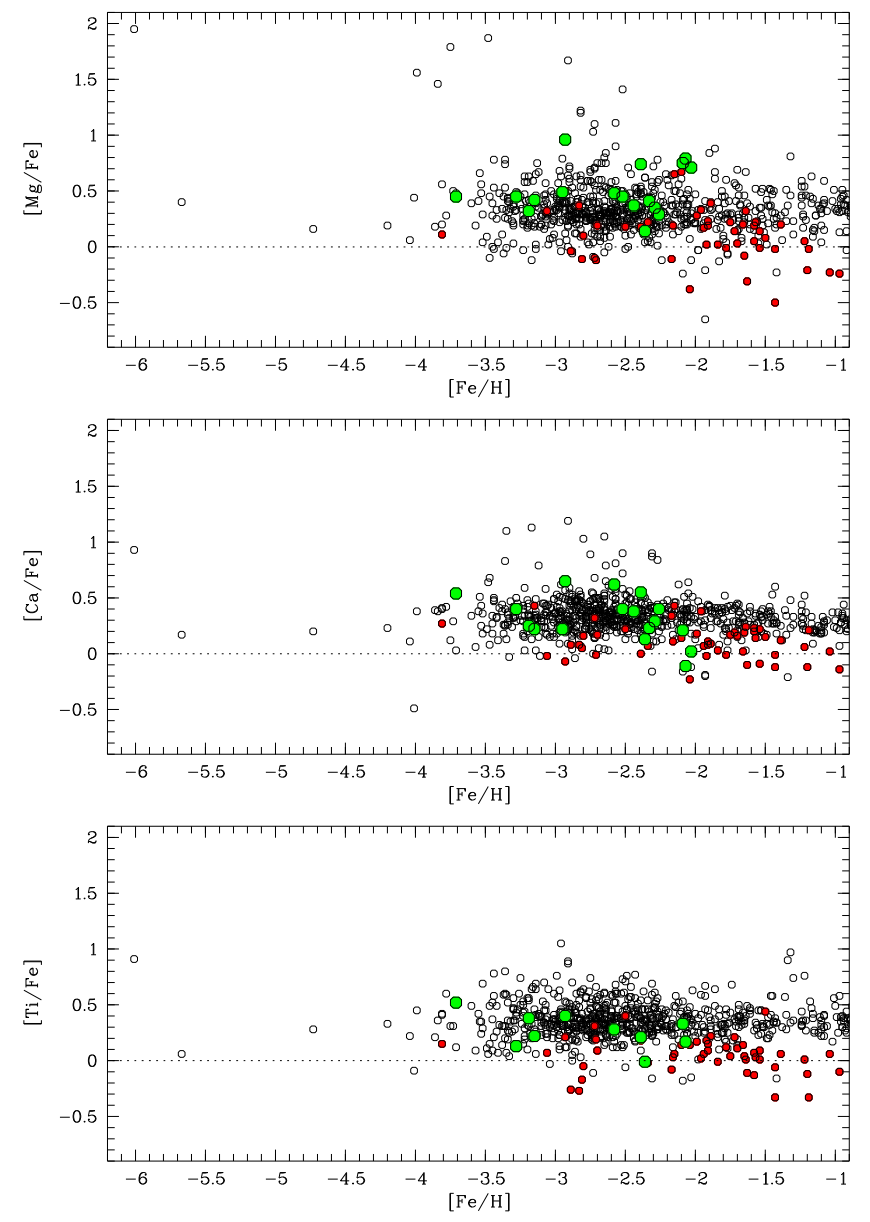

Fig. 4 Light element abundance trends of $\mathrm{Mg}, \mathrm{Ca}$, and Ti. Black open circles represent halo stars, red filled circles are stars in the classical dwarf galaxies, and green filled circles show stars in the ultra-faint dwarf galaxies. The abundance data can be found in an electronic format. The scatter in the data likely reflects systematic differences between literature studies. Assuming this, systematic uncertainties in abundance analyses may be around $\sim 0.3$ dex. Figure taken from Frebel 2010.

the solar value at $[\mathrm{Fe} / \mathrm{H}] \sim 0.0$. The general uniformity of light element abundance trends down to $[\mathrm{Fe} / \mathrm{H}] \sim-4.0$ led to the conclusion that the ISM must have been already well-mixed at very early times (Cayrel et al. 2004). Otherwise it would be hard to understand why so many of the most metal-poor stars have almost identical abundance patterns. However, despite the well-defined abundance trends, some stars, particularly those in the lowest metallicity regime show significant deviations. Among those are some stars with unusually high or low $\alpha$-element abundances.

Among the Fe-peak elements, many have subsolar abundance trends at low metallicity (e.g. $[\mathrm{Cr}, \mathrm{Mn} / \mathrm{Fe}])$ which become solar-like as the metallicity increases. 

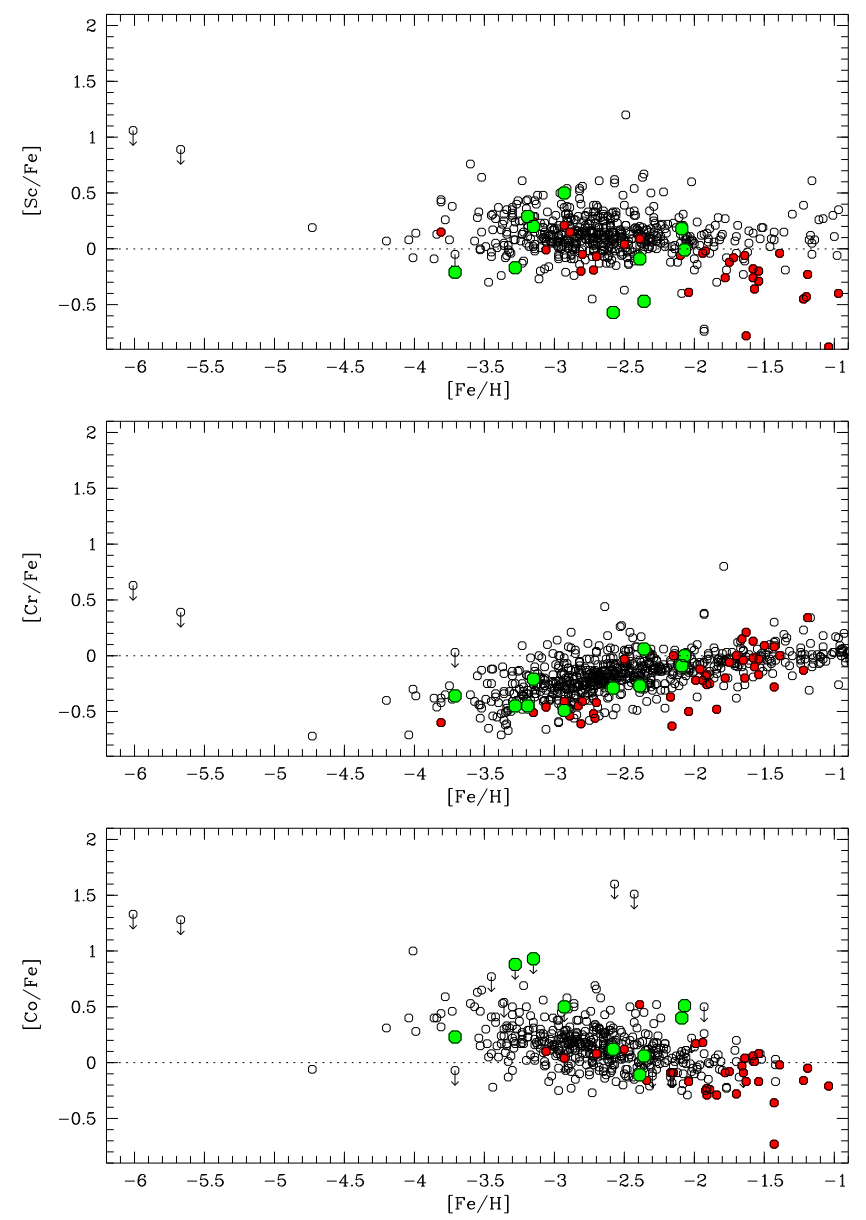

Fig. 5 Same as Figure 4 but for the Fe-peak elements Sc, Cr, and Co. Figure taken from Frebel 2010.

This is shown in Figure 5 It is not clear whether these large underabundances are of cosmic origin or have to be attributed to modeling effects such as that of non-LTE (Sobeck et al. 2007; Bergemann et al. 2008). Trends of other elements are somewhat overabundant at low metallicity (Co) or relatively unchanged throughout ( $\mathrm{Sc}, \mathrm{Ni}$ ). All elements with $Z<30$ hereby have relatively tight abundance trends.

On the contrary, the abundances of the neutron-capture elements in metal-poor stars are "all over the place". Sr has an extremely large scatter $(\sim 3 \mathrm{dex})$. This indicates that different nucleosynthetic processes must have contributed to its Galactic inventory, or that neutron-capture yields are very environmentally-sensitive. Ba has even more scatter at $[\mathrm{Fe} / \mathrm{H}] \sim-3.0$ (see Figure 6). Some of this spread may be explained through the existence of massive rotating low-metallicity stars that produce large amounts of s-process like $\mathrm{Sr}$ and $\mathrm{Ba}$ in the early universe (Chiappini 

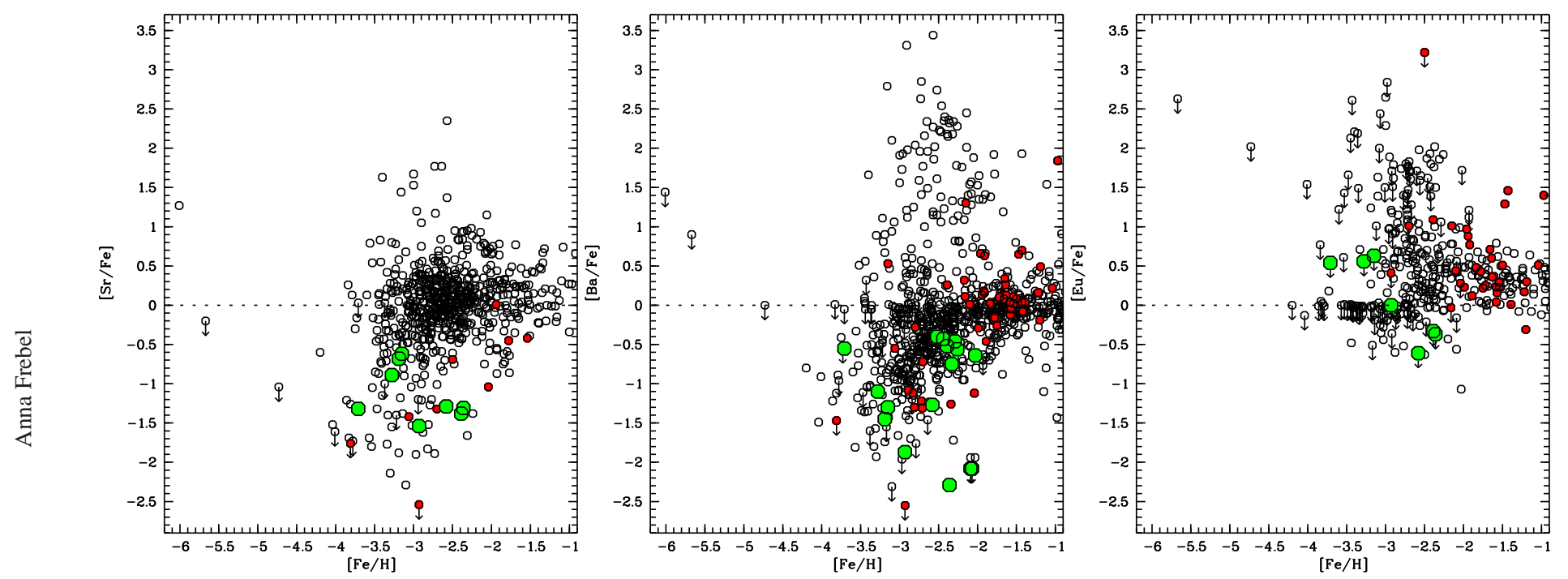

Fig. 6 Same as Figure 4 but for the neutron-capture elements Sr, Ba, and Eu. For these elements, the scatter is much beyond systematic differences between individual studies and thus indicates a cosmic origin. Figure taken from Frebel 2010. 
et al. 2011). Other heavier neutron-capture elements, such as r-process element Eu, have somewhat less scatter. At the lowest metallicities, core-collapse SNe must have dominated the chemical evolution (below $[\mathrm{Fe} / \mathrm{H}]=-3.0$ ). Hence, the r-process is likely responsible for the neutron-capture elements at this early time. Metal enrichment through the s-process began to significantly contribute at somewhat later times, driven by the evolutionary timescales of stars with $\sim 2-8 \mathrm{M}_{\odot}$ to become AGB stars. According to Simmerer et al. (2004), the s-process was in full operation at $[\mathrm{Fe} / \mathrm{H}] \sim-2.6$.

\subsection{Tracing individual SN explosions with the most iron-poor stars}

The faint $(V=15.2)$ red giant HE $0107-5240$ has $[\mathrm{Fe} / \mathrm{H}]=-5.2($ Christlieb et al. 2002). The brighter $(V=13.5)$ subgiant HE $1327-2326$ has an even lower iron abundance of $[\mathrm{Fe} / \mathrm{H}]=-5.4$ (Frebel et al. 2005; Aoki et al. 2006). The latter value corresponds to $\sim 1 / 250,000$ of the solar iron abundance 4 A third star with $[\mathrm{Fe} / \mathrm{H}]=-4.75$ (Norris et al. 2007) was found in 2006. The metallicity of the giant HE $0557-4840$ is in between the two $[\mathrm{Fe} / \mathrm{H}]<-5.0$ stars and the next most metal-poor stars are $[\mathrm{Fe} / \mathrm{H}] \sim-4.0$. Hence, it sits right in the previously claimed "metallicity gap" (between $[\mathrm{Fe} / \mathrm{H}] \sim-4.0$ and $[\mathrm{Fe} / \mathrm{H}] \sim-5.0$; e.g. Shigeyama et al. 2003) showing that the scarcity of stars below $[\mathrm{Fe} / \mathrm{H}]=-4.0$ has no physical cause but is merely an observational incompleteness. All three objects were found in the Hamburg/ESO survey making it the so far most successful database for metalpoor stars.

The most striking features in both $[\mathrm{Fe} / \mathrm{H}]<-5.0$ stars are the extremely large overabundances of CNO elements. HE 0557-4840 partly shares this signature by also having a fairly large $[\mathrm{C} / \mathrm{Fe}]$ ratio. Other elemental ratios $[\mathrm{X} / \mathrm{Fe}]$ are somewhat enhanced in $\mathrm{HE} 1327-2327$ with respect to the stars with $-4.0<[\mathrm{Fe} / \mathrm{H}]<-2.5$, but less so for the two giants. Despite expectations, Li could not be detected in the relatively unevolved subgiant HE $1327-23265$ The upper limit is $\log \varepsilon(\mathrm{Li})<1.6$, where $\log \varepsilon(\mathrm{A})=\log _{10}\left(N_{\mathrm{A}} / N_{\mathrm{H}}\right)+12$. This is surprising, given that the primordial Li abundance is often inferred from similarly unevolved metal-poor stars (Ryan et al. 1999). Furthermore, the upper limit found from HE 1327-2326, however, strongly contradicts the WMAP value $(\log \varepsilon(\mathrm{Li})=2.6)$ from the baryon-to-photon ratio (Spergel et al. 2007). This may indicates that the star formed from extremely Li-poor material. No neutron-capture element is detected in HE 0107-5240 or HE 0557-4840, whereas, unexpectedly, $\mathrm{Sr}$ is observed in HE 1327-2326. Massive rotating stars may be responsible for this neutron-capture element (Chiappini et al. 2011).

\footnotetext{
${ }^{4}$ Interestingly, the entire mass of iron in HE 1327-2326 is actually 100 times less than that in the Earth's core. At the same time the star is of course of the order of a million times more massive than the Earth.

${ }^{5}$ The other stars are giants. Thus, the surface $\mathrm{Li}$ is already destroyed due to the thick convection zone transporting Li to deeper, hotter layers where it burns.
} 
How can all those signatures be understood in terms of early chemical enrichment? HE 0107-5240 and HE 1327-2326 immediately became benchmark objects to constrain various theoretical studies of the early Universe, such as the formation of the first stars (e.g., Yoshida et al. 2006), the chemical evolution of the early ISM (e.g., Karlsson \& Gustafsson 2005) or calculations of Pop III SN yields. Their highly individual abundance patterns have been successfully reproduced by several different SNe scenarios. This makes HE 0107-5240 and HE 1327-2326 early, extreme Pop II stars that possibly display the "fingerprint" of just one Pop III SN. Umeda \& Nomoto (2003) first matched the yields of a faint $25 \mathrm{M}_{\odot}$ SN that underwent a mixing and fallback process to the observed abundances of HE 0107-5240. To achieve a simultaneous enrichment of a lot of $\mathrm{C}$ and only little Fe, large parts of the Fe-rich SN ejecta have to fall back onto the newly created black hole. Using yields from a SN with similar explosion energy and mass cut, Iwamoto et al. 2005 then reproduced the abundance pattern of HE 1327-2326 also. Trying to fit the observed stellar abundances, Heger \& Woosley (2010) are employing an entire grid of Pop III SN yields to search for the best match to the data. A similar progenitor mass range as the Umeda \& Nomoto (2003) $25 \mathrm{M}_{\odot}$ was found to be the best match to have provided the elemental abundances to the ISM from which these Pop II stars formed.

Limongi et al. (2003) were able to reproduce the abundances of HE 0107-5240 through pollution of the birth cloud by at least two SNe. Suda et al. (2004) proposed that the abundances of HE 0107-5240 would originate from a mass transfer of CNO elements from a postulated companion, and from accretion of heavy elements from the ISM. However, neither HE 0107-5240 nor HE 1327-2326 show radial velocity variations that would indicate binarity. Meynet et al. (2005) explored the influence of stellar rotation on elemental yields of $60 \mathrm{M}_{\odot}$ near-zero-metallicity SNe. The stellar mass loss rate of rotating massive Pop III stars qualitatively reproduces the CNO abundances observed in HE 1327-2326 and other carbon-rich metal-poor stars.

More generally, the observed abundances of the most metal-poor stars with typical halo signatures in the range have successfully been reproduced with Pop III $\mathrm{SN}$ yields. Tominaga et al. (2007b) model the averaged abundance pattern of four non-carbon-enriched stars with $-4.2<[\mathrm{Fe} / \mathrm{H}]<-3.5$ with the elemental yields of a massive, energetic $\left(\sim 30-50 \mathrm{M}_{\odot}\right)$ Pop III hypernova. The abundances can also be fitted with integrated yields of Pop III SNe (Heger \& Woosley 2010). Special types of $\mathrm{SNe}$ or unusual nucleosynthesis yields can then be considered for stars with chemically peculiar abundance patterns. It is, however, often difficult to completely explain the entire abundance pattern of a given star. Additional metal-poor stars as well as a better understanding of nucleosynthesis and the explosion mechanism and the impact of the initial conditions on SNe yields are required to arrive at a more solid picture of the details of early $\mathrm{SNe}$ nucleosynthesis. 


\section{Dwarf Archaeology}

Simulations of the hierarchical assembly of galaxies within the cold dark matter (CDM) framework (Diemand et al. 2007; Springel 2005) show that the Milky Way halo was successively built up from small dark matter substructures, often referred to as galactic building blocks, as long ago suggested by Searle \& Zinn (1978). Figure 7 shows the substructure around the six simulated, high-resolution "Aquarius" halos at $z=0$ (Springel et al. 2008; Lunnan et al. 2011). The satellites around the main halos are clearly visible - these are smaller halos that survived the violent accretion process until today 6 They can be regarded as the counterparts to today's satellite population of the Milky Way.

However, these simulations generally only include dark matter, and it remains unclear to what extent small dark halos contain luminous matter in the form of stars and gas. This question is particularly important with respect to the so-called "missing-satellite" problem which reflects the mismatch of the number of observed dwarf galaxies surrounding the Milky with the predicted number of substructures for a Milky Way-like halo. Studying the onset of star formation and associated chemical evolution in dwarf galaxies thus provides some of the currently missing information for our understanding of how the observed properties of small satellites relate to the (dark matter) substructures that build up larger galaxies. Thus, the study of the entire stellar population of a dwarf galaxy for the purpose of inferring details about the nature and origin of the first galaxies and early galaxy assembly is termed "dwarf archaeology".

\subsection{Chemical Evolution of Dwarf Galaxies}

The connection between the surviving dwarfs and those that dissolved to form the halo can partially be addressed by examining in detail the stellar chemical compositions of present-day dwarf galaxies. Establishing detailed chemical histories of these systems can provide constraints on their dominant chemical enrichment events and timescales. From such information, conclusions about the formation process of the Milky Way can be derive. Specifically, detailed knowledge of the most metalpoor stars in a given system allow insight into its earliest phases of star formation before the effects of internal chemical evolution were imprinted in stars born later with higher metallicity. Despite the fact that dwarf galaxies are regarded as "simple" systems, many of them show extended star formation histories with similar, albeit slower, chemical evolution to the Milky Way (for further details on this topic the reader is referred to the recent reviews by Tolstoy et al. 2009 and Koch 2009). Altogether, they are old, gas-less systems, and a tight correlation between the average metallicity and systemic luminosity.

${ }^{6}$ In this simulation, prescriptions for the population of dark halos with luminous matter have already been applied. See Lunnan et al. (2011) for further details and references therein. 

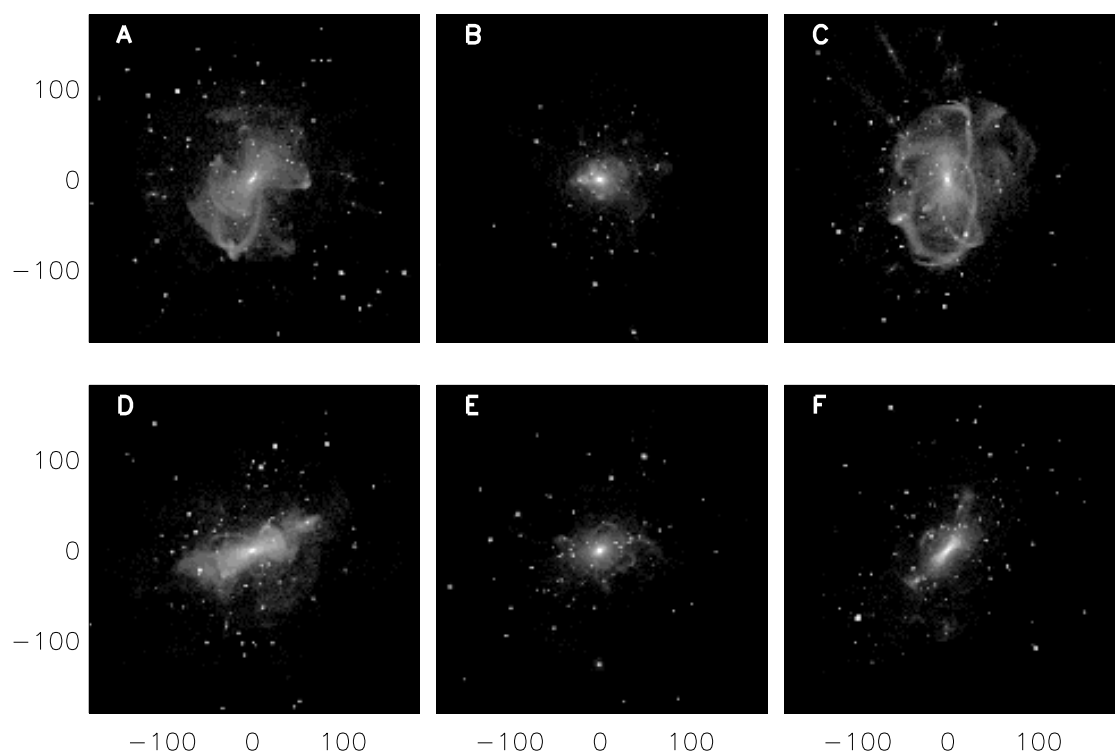

Fig. 7 Projected spatial distribution of surviving substructure, as well as accreted material that forms a stellar halo, in the six Aquarius simulations. The scale is in kpc. Figure taken from Lunnan et al. (2011).

Kirby et al. (2008) extended the metallicity-luminosity relationship to the ultrafaint $\left(L<10^{5} L_{\odot}\right.$; discovered in the Sloan Digital Sky Survey; SDSS) dwarfs. They also showed that the lowest luminosity dwarfs have very low average metallicities (down to $<[\mathrm{Fe} / \mathrm{H}]>\sim-2.6$ ), with several systems having values lower than those of the most metal-poor globular clusters. Many individual stars even have $[\mathrm{Fe} / \mathrm{H}]<$ -3 , while interestingly, no stars with $[\mathrm{Fe} / \mathrm{H}]>-1.0$ are found. The combined MDF of all the ultra-faint dwarfs extends down to $[\mathrm{Fe} / \mathrm{H}] \sim-3.8$, and the shape appears similar to that of the Milky Way halo (for the low-metallicity tail, that is), although perhaps with a slightly larger relative fraction towards the most metal-poor stars. Thus, it is not surprising that the ultra-faint dwarf galaxies contain a relatively many of extremely metal-poor stars, with large internal $[\mathrm{Fe} / \mathrm{H}]$ abundance spreads of up to 3 dex. These spreads were first found in lower spectral resolution data (e.g., Simon \& Geha 2007; Norris et al. 2008) and later confirmed with high-resolution follow-up of individual stars. At the same time, it became apparent that $\sim 30 \%$ of the known stars with $[\mathrm{Fe} / \mathrm{H}]<-3.5$ are now found in dwarf galaxies. Segue 1, the most metalpoor system, contains $\sim 15 \%$ alone (Frebel et al. in prep.).

Such high-resolution spectra are needed for a full chemical abundance analysis. Given the faint magnitude of stars in dwarf galaxies, these data are difficult to acquire, but good progress has been made over the past few years. In fact, most stars with $V<19.2$ available in the ultra-faint dwarfs have now been observed this way. 
More ultra-faint dwarfs are expected to be found soon in surveys such as Pan-Starrs, Skymapper and LSST, thus providing new targets that can again be observed with high-resolution on current 6-10 m telescopes.

Generally, these kinds of data enable measurements of chemical abundances and upper limits of 10-15 elements for a given star. For example, the three brightest stars in each of Ursa Major II (UMa II) and Coma Berenices (ComBer) and two stars in Hercules are the first stars in the ultra-faint dwarf galaxies to have been observed with high-resolution spectroscopy. Two of them (in UMa II) are also the first known extremely metal-poor stars which are not members of the halo field population. Details on the observations and analysis techniques are given in Frebel et al. (2010a) and Koch et al. (2008). For the UMa II and ComBer stars, chemical abundances and upper limits of up to 26 elements were determined for each star. The abundance results demonstrate that the evolution of the elements in the ultra-faint dwarfs is very similar to that of the Milky Way, and likely dominated by massive stars. The $\alpha$-elements in these two ultra-faint dwarf stars are overabundant, showing the halo-typical core-collapse $\mathrm{SNe}$ signature (see Figure 4 ).

The neutron-capture abundances are extremely low in the ultra-faint dwarf stars (see green (large shaded) circles in Figure 6). The observed $\mathrm{Sr}$ and Ba values are up to two orders of magnitude below the abundances found in typical MW halo stars with similar Fe content. However, a very large spread (up to 3 dex) in these elements is found among halo stars themselves. The large depletions in the dwarf galaxy stars are thus not inconsistent with the halo data since similarly low values are found in numerous objects. Interestingly though, the low neutron-capture abundances may represent a typical signature of stars in dwarf galaxies. Similarly low values are also found in Hercules (Koch et al. 2008) and Draco (Fulbright et al. 2004) despite their sometimes relatively high $\mathrm{Fe}$ values of $[\mathrm{Fe} / \mathrm{H}] \sim-2.0$ (in Hercules).

By applying improved search techniques also to the more luminous dwarf galaxies, the first extremely metal-poor star in a classical dwarfs Sculptor was recently discovered (in a sample of 380 stars; Kirby et al. 2009). The metallicity of $[\mathrm{Fe} / \mathrm{H}] \sim$ -3.8 was confirmed from a high-resolution spectrum taken with Magellan/MIKE (Frebel et al. 2010b). Only nine stars in the halo have even lower Fe abundances than this object. Shortly afterwards, Tafelmeyer et al. (2010) presented additional, similarly metal-poor stars in Sculptor, and also Fornax. These discoveries show that such low-metallicity stars are indeed present in the more luminous dwarfs (see also Starkenburg et al. 2010), contrary to earlier claims.

The new stars underpin that metallicity spreads of $\sim 3$ dex or more are present in many classical dwarfs. The chemical abundances obtained from the high-resolution spectrum reveal a similar picture to what has been found in the ultra-faint dwarf stars. For example, the Sculptor star at $[\mathrm{Fe} / \mathrm{H}] \sim-3.8$, also has a remarkably similar chemical make-up compared to that of the Milky Way halo at the lowest metallicities. This is in contrast to what is found at higher metallicities in these brighter dwarfs. As can be seen in Figure 4 (red (small shaded) circles), those stars have lower stellar $\alpha$-abundance ratios than comparable halo stars, indicating different enrichment mechanisms and timescales in these gas-poor systems (e.g., Shetrone et al. 2003; Geisler et al. 2005). 


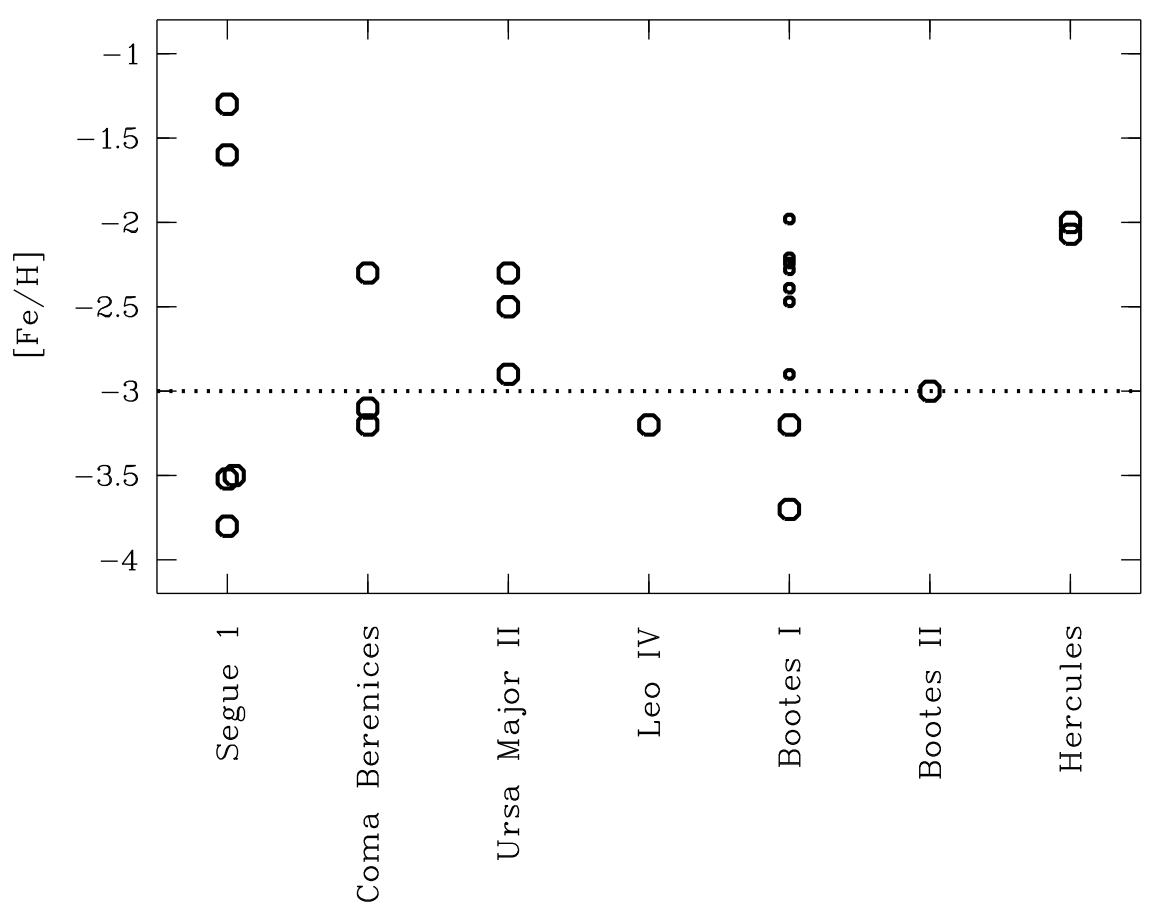

Fig. 8 Current status of available high-resolution spectroscopy of stars in the ultra-faint dwarf galaxies. Many stars have $[\mathrm{Fe} / \mathrm{H}]<-3.0$ Large circles indicate extensive abundances studies of many elements, whereas smaller circles refer to studies of only a few elements. Data from Koch et al. (2008), Feltzing et al. (2009), Frebel et al. (2010a), Simon et al. (2010), Norris (2010b), Norris et al. (2010a) and Frebel et al. (2011, in prep.)

\subsection{Individual Pop III Enrichments?}

The large number of extremely metal-poor stars in the faintest dwarf galaxies is very surprising given the very limited total stellar material in these systems with $L_{t o t} \lesssim 10^{5} L_{\odot}$. This represents an opportunity to study the environment of a lowmetallicity galaxy which should have hosted only a few SNe. Consequently, signs of individual, stochastic enrichment events may be preserved in the metal-poor stars.

Enrichment by a single $13 \mathrm{M}_{\odot}$ has been suggested for the Hercules dwarf $\left(L \sim 10^{5} L_{\odot}\right)$, based on the chemical abundances of two stars with $[\mathrm{Fe} / \mathrm{H}] \sim-2.0$ and unusually low levels of Ba (Koch et al. 2008). With its low luminosity of $\left(L_{V}=14,000 L_{\odot}\right)$ and low metallicity, Simon et al. (2010) determined that Leo IV contains only $0.042 \mathrm{M}_{\odot}$ of Fe (assuming a stellar mass-to-light ratio of $1 \mathrm{M}_{\odot} / \mathrm{L} \odot$ ). Canonical SN nucleosynthesis yield calculations predict a maximum Fe yield of $\sim 0.1 M_{\odot}$ (e.g. Heger \& Woosley 2010). The total Fe amount in Leo IV, and by extension that of all the other observed elements, could thus stem from a single star that exploded soon after the system formed. The star forming gas cloud at that 

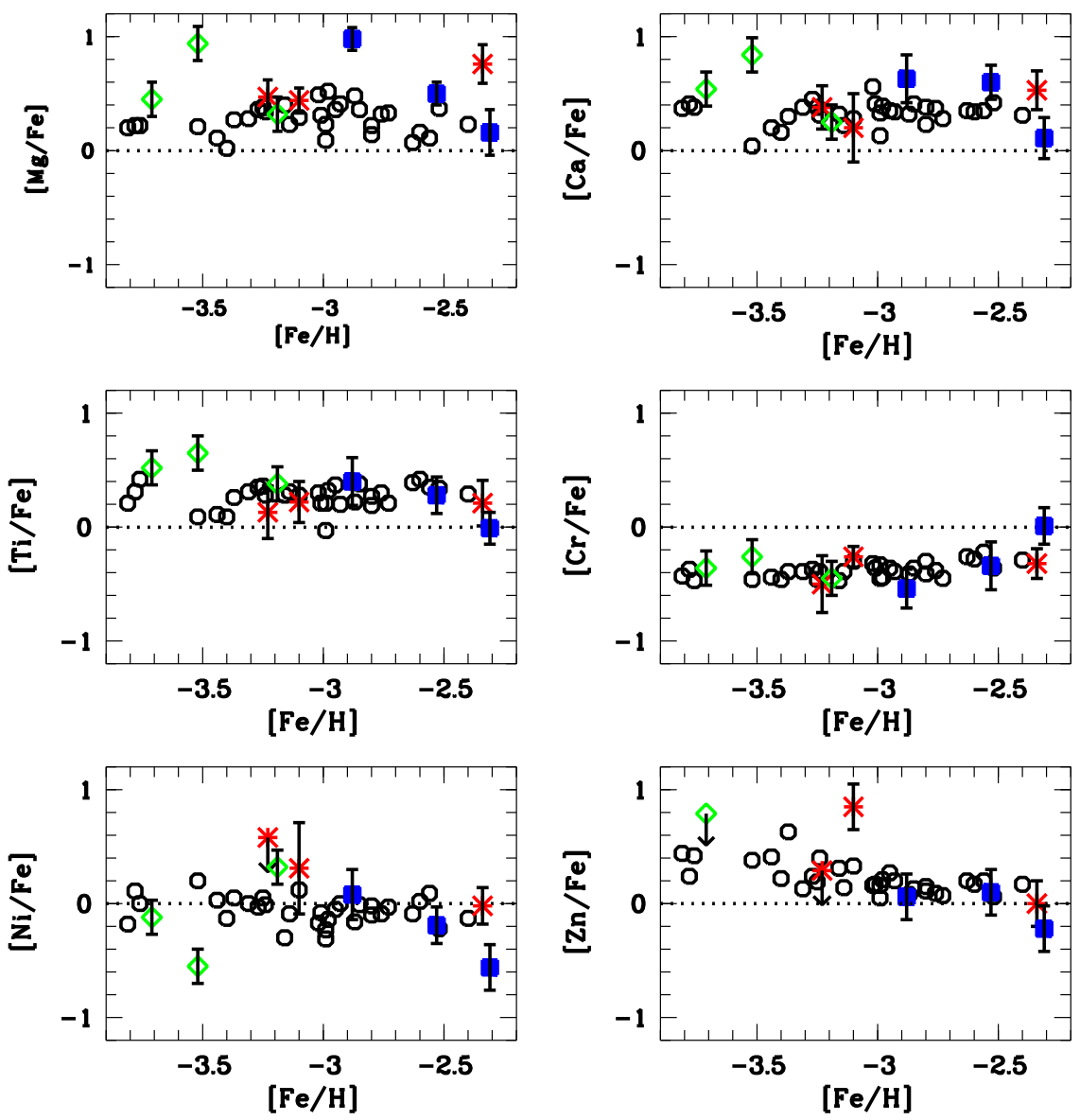

Fig. 9 Chemical abundance comparison of Galactic halo stars (open circles, from Cayrel et al. 2004) and stars in the ultra-faint dwarfs (asterisks: Ursa Major II, filled squares: Coma Berenices, diamonds: Segue 1, Bootes I and Leo IV). The abundances generally agree very well, suggesting a similar enrichment history of the gas from which all these stars formed. Based on Figure 20 from Frebel \& Norris (2011).

time must have been only $\sim 40,000 M_{\odot}$. Given that the chemical abundances of the brightest star that is accessible to high-resolution spectroscopic studies, resemble those of metal-poor halo stars, it was suggested that indeed a single Pop III star was responsible for Leo IV's enrichment. Various SN nucleosynthesis models invoking different progenitor masses and explosion energies (e.g., Tominaga et al. 2007b; Heger \& Woosley 2010) have shown that the typical halo abundance signature can be explained this way.

Alternatively, given the shallow potential of these kinds of systems, several $\mathrm{SNe}$ could have contributed to the enrichment, but part of the enriched gas was soon lost (e.g., through stellar winds or SN explosions). Future observations of stars in ultra- 
faint dwarfs will provide more detailed insight. Nevertheless, the currently available data already suggest that these systems played an important role for our understanding of the first stars and chemical enrichment events that shaped the nature of these relatively small, early systems.

\subsection{Are the faintest dwarf satellites surviving first galaxies?}

Given that the ultra-faint dwarf galaxies are the most metal-poor objects we know of today, they are ideal probes of the physical, chemical, and dynamical processes at work in the early universe. Since these systems have much simpler star formation histories than the halo of the Milky Way, their stellar populations should preserve the fossil record of the first supernova (SN) explosions in their long-lived, lowmass stars (see also Section 4.2). Hence, the metal-poor stars in the ultra-faint dwarf galaxies should be used to empirically constrain the formation process of the first galaxies, and early galaxy assembly more generally.

Guided by recent hydrodynamical cosmological "ab-initio"simulations (Greif et al. 2008; Greif et al. 2010) of first galaxy formation, Frebel \& Bromm (2011) developed a set of stellar abundance signatures that characterizes the nucleosynthetic history of such an early system if it was observed in the present-day universe. In particular, the simulations suggest that a first galaxy can be regarded a chemical "one-shot" event, where only one (long-lived) stellar generation forms after the first, Population III, SN explosions. The system would thus be dominated by an $[\alpha / \mathrm{Fe}]$ enrichment due to enrichment by massive stars as seen in the halo at low metallicity. With no stars present displaying an erstwhile enrichment by AGB stars of SN Ia, the $\alpha$-enhancement would also be present in stars with higher metallicities $([\mathrm{Fe} / \mathrm{H}]>-1.5)$. These criteria thus constrain the strength of negative feedback effects inside the first galaxies.

A comparison of the abundances of about a dozen stars in the ultra-faint dwarfs with this one-shot criterion indicates that some of these faintest satellites could be surviving first galaxies. Several systems (Ursa Major II, and also Coma Berenices, Bootes I, Leo IV, Segue 1) largely fulfill the requirements (most notably the high $\alpha /$ Fe ratios), indicating that their high-redshift predecessors did experience strong feedback effects that shut off star formation soon after the formation of the system. More observations are needed to firm up these initial conclusions, and also additional simulations of early metal mixing, turbulence and the extend of feedback in early, assembling galaxies. This will provide clues to the connection of the first galaxies, the surviving, metal-poor dwarf galaxies, and the building blocks of the Milky Way. 


\section{Near-field cosmology}

A long-standing problem, originally noted by e.g. Moore et al. (1999) and Klypin et al. (1999), is that the observed number of Milky Way satellites appears to be significantly lower than the number of dark matter substructures expected based on the CDM theory (the so-called "missing satellite problem"). This calls into question the validity of the CDM picture on the scales of individual galaxies. Many ideas have been proposed to reconcile the theory with the observations, including the possibility that the dark matter may instead be dynamically "warm", rather than cold (achieved by making the individual dark matter particles less massive). Another class of models proposes instead that effects related to feedback from baryonic processes or heating by cosmic radiation fields may inhibit star formation in dark matter halos of sufficiently low mass, rendering them invisible. Either way, a definitive solution to the missing satellite problem will inform our theories of the nature of the dark matter and the assembly of galaxies.

Progress has been made through detailed studies of different types of dwarf galaxies (ultra-faint, classical dwarf spheroidal, more massive dwarfs such as the Magellanic Clouds) which orbit the Milky Way. Extensive photometric and spectroscopic data of these satellites, paired with the discovery of stellar streams in the Galactic halo arising from past and even ongoing mergers of the massive host with smaller galaxies, have revealed much about the complex nature of the life and times of dwarf galaxies and their role in shaping their parent galaxy. Figure 10 shows a number of streams that are currently present on the stellar halo of the Milky Way due to past accretion events.

Nevertheless it is surprising that the Milky Way overall has so few satellite galaxies when its sister galaxy Andromeda appears to have a significantly larger population signaling a potentially very different assembly history. By learning about the Galaxy and its assembly, as well as pushing for large-scale simulations to address cosmic variance and where the Milky Way really fits within the zoo of galaxies, we will soon be able to quantify the host of observations that suggest the unique nature of our Galaxy.

\subsection{Did the stellar halo form from accreted dwarf galaxies?}

The overarching questions of near-field cosmology concern the nature of the "building blocks" of large galaxies, and to what extent dwarf galaxies play a role in the assembly of old stellar halos. This comes at a time when in particular the population of Milky Way ultra-faint dwarf galaxies have been shown to be extremely metaldeficient systems that host $\sim 30 \%$ of the known most metal-poor stars. Moreover, they extend the metallicity-luminosity relationship of the classical dwarfs down to $L_{\text {tot }} \sim 10^{3} L_{\odot}$ (see Kirby et al. 2008 for more details). Future observations will reveal how far this relationship can be extended. 


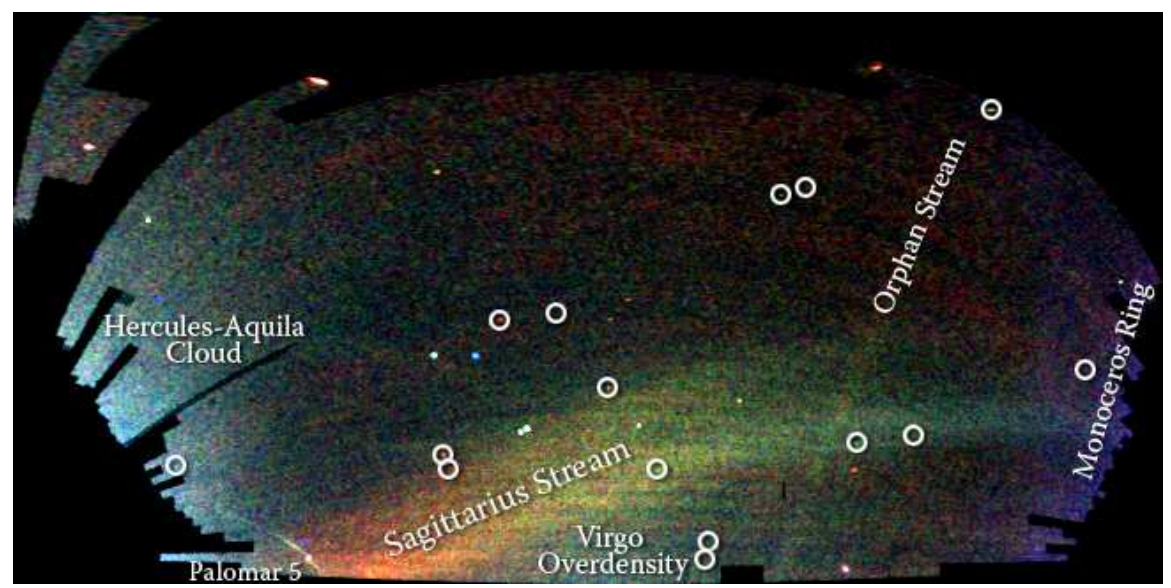

Fig. 10 Field of Streams: A map of stars in the outer regions of the Milky Way Galaxy, shown in a Mercator-like projection. The color indicates the distance of the stars, while the intensity indicates the density of stars on the sky. Structures visible in this map include streams of stars torn from the Sagittarius dwarf galaxy, a smaller "orphan" stream crossing the Sagittarius stream, the "Monoceros Ring" that encircles the Milky Way disk, trails of stars being stripped from the globular cluster Palomar 5, and excesses of stars found towards the constellations Virgo and Hercules. Circles enclose new Milky Way companions discovered by the SDSS; two of these are faint globular star clusters, while the others are faint dwarf galaxies. Image credit: V. Belokurov and the Sloan Digital Sky Survey.

If the old, metal-poor halo was indeed assembled from dwarf galaxies, the metallicities of stars in dwarf galaxies must reach values as low as (or lower) what is currently found in the Galactic halo population. Moreover, the abundance ratio of those low-metallicity stars must be roughly equal to those of equivalent stars in the halo.

Earlier works missed finding extremely metal-poor stars in the classical dwarfs, which posed a significantly problem to the idea of an accreted halo through systems similar to the surviving dwarfs. However, it has now been shown that this claim stems merely from biases in earlier search techniques (Cohen et al. 2009; Kirby et al. 2008a; Starkenburg et al. 2010; Kirby et al. 2010). With improved methods for identifying the lowest-metallicity objects, extremely metal-poor stars have already been identified in several dwarf galaxies (Kirby et al. 2008; Geha et al. 2009; Frebel et al. 2010b; Tafelmeyer et al. 2010). The higher-metallicity stars in the classical dwarfs all have abundance ratios different from comparable halo stars. Most strikingly, the $\alpha$-element abundances are not enhanced, which must be due to the onset on SN Ia enrichment at a time when the overall chemical evolution of these small systems was less evolved than the halo. It also reflects that any of these stars are the product of the galaxy's own chemical evolution that has nothing in common with that of the Milky Way. However, there is increasing evidence that a transition to more halo-typical abundance ratios may take place around a metallicity of $[\mathrm{Fe} / \mathrm{H}]=-3.0$ (Cohen et al. 2009; Aoki et al. 2009) in these dwarfs. This means that if the halo formed from accreted satellites, any systems like the classical dwarfs 
must have been accreted at a relatively early time when the chemical evolution had not much progressed. Given that the dwarfs we observe today were not accreted, they kept forming stars and continued chemical evolution until they lost all their gas.

Due to their simple nature, the ultra-faint systems are expected to retain signatures of the earliest stages of chemical enrichment in their stellar populations. If the halo was assembled from early analogs of the surviving dwarfs, most of the metal-poor halo stars should have been deposited there during late-time accretion events (Frebel et al. 2010a; Simon et al. 2010; Font et al. 2006). Indeed the chemical abundances of individual stars in the faintest galaxies suggest a close connection to equivalent, extremely metal-poor halo stars in the Galaxy.

The recent high-resolution studies (Feltzing et al. 2009; Frebel et al. 2010a; Norris et al. 2010a,2010b; Simon et al. 2010) provide evidence that the abundance patterns of light elements $(Z<30)$ in these dwarfs are remarkably similar to the Milky Way halo, even for stars with $[\mathrm{Fe} / \mathrm{H}]>-3.0$. this is illustrated in Figure 9 which shows a very detailed comparison between halo and ultra-faint dwarf galaxy stars. The similarity between the data sets is clearly seen. However, given the limited number of stars it is still unclear up to what metallicity the halo-typical abundances are found in these systems. There are indications that the chemical evolution in the ultra-faint dwarfs may have been very inhomogeneous, and also, that a number of stars show strong carbon-enhancement (Norris et al. 2010a). Taken altogether, these features are found among the lowest metallicity halo stars, making a plausible case for an accretion-built halo, at least for stars with $[\mathrm{Fe} / \mathrm{H}]<-2.5$.

These observational results about the halo assembly are broadly consistent with the predictions of CDM cosmological models (e.g. Robertson et al. 2005; Johnston et al. 2008). While the majority of the mass that formed the inner part of the stellar halo (at $[\mathrm{Fe} / \mathrm{H}] \sim-1.2$ to -1.6$)$ was provided at early times by much larger systems such as the Magellanic Clouds, the outer halo assembled at later times. In fact, as shown in Figure 9, it is still assembling today, with ongoing accretion event leading to a variety of streams and substructure. The similarity of the chemical abundances, suggesting the same chemical enrichment history in these stars prior to their formation, make it plausible to assume that the ultra-faint dwarf galaxies contributed (some) individual metal-poor stars to the Galaxy. These stars are now found primarily in the outer Galactic halo.

However, these systems may not have been sufficiently numerous to account for the entire metal-poor end of the Fe metallicity distribution of the Milky Way halo. Since the classical dSphs have more stellar mass and have been shown to also contain at least some of the most metal-poor stars (e.g., Kirby et al. 2009; Frebel et al. 2010b), they could have been a major source of the lowest-metallicity halo stars. Additional observations of more extremely metal-poor stars in the various dwarf galaxies are highly desirable in the quest to determine individual MDFs for each of these galaxies, and how those would compare with each other and that of the Milky Way. 


\subsection{Towards constraining the reionization history of the Milky Way}

Cosmological simulations of the growth of structure have shown that galaxy assembly proceeds hierarchically. The results of these studies have indicated a mismatch between the predicted number of low-mass, dark matter substructures and the actual observed number of satellite galaxies around the Milky Way; the "missing satellite problem". The underlying cause of this discrepancy can be investigated with cosmological simulations. By examining the impact of various physical processes on the evolution of faint galaxies, the nature of the surviving luminous satellites and their stellar content can be understood in more detail. An important question is this regard is which effects critically determine the number of small satellites hosting luminous matter throughout the build-up of a large galaxy, and whether there would be enough of them to form a metal-poor stellar halo of their host, similar to what is found for the Milky Way.

One explanation for the small number of faint satellites is that as the universe reionizes, the increased temperature of the IGM prevents the smallest halos from accreting and cooling gas, preventing further star formation. In this scenario, the nature and abundance of the faintest dwarf galaxies can in principle provide a constraint on the reionization history of the Milky Way (Munoz et al 2009, Busha et al. 2010, Lunnan et al. 2011). This is illustrated in Figure 11 which shows observed and predicted luminosity functions of dwarf galaxies for the six Milky Way-like halos from the Aquarius simulation, combined with four different reionization models. The dotted line shows the prediction with no reionization effects, clearly illustrating the "missing satellite" problem. This is the case for all six halos. The general haloto-halo scatter (factor of 2-3; see also Figure 7), however, suggests that the missing satellite problem is in part due to cosmic variance. Moreover, taking the effects of reionization into account has a pronounced impact of just the faintest halos. While larger halos (equivalents to today's brighter dwarf galaxies) are relatively insensitive to a non-instantaneous reionization prescription, the number of surviving small galaxies changes significantly with the reionization model for different mean reionization redshift and Thompson optical depths still in agreement with the WMAP value (Komatsu et al. 2011).

Given these initial results, it appears promising to use the brighter end of the luminosity function to constrain the halo-to-halo scatter, while the faint end will enable to discriminate reionization histories. However, a solid understanding of how "normal" the Milky Way is and where falls within these halo-to-halo variations and will be required. Future simulations may be able to quantify the nature and being of the Galaxy with respect to the majority of other large galaxies. Knowing the substructure abundance of galaxy halos is critical for interpreting observations of the satellite populations of all large galaxies, including the Milky Way and Andromeda. Moreover, estimates would become possible of the stellar contribution to the halo as a function of halo mass and the associated merger and accretion history.

Altogether, the opportunity to constrain the reionization history of the Milky Way through a careful analyses of the faintest satellite population will help understanding the nature of these systems themselves. Knowing the fraction of surviving small 


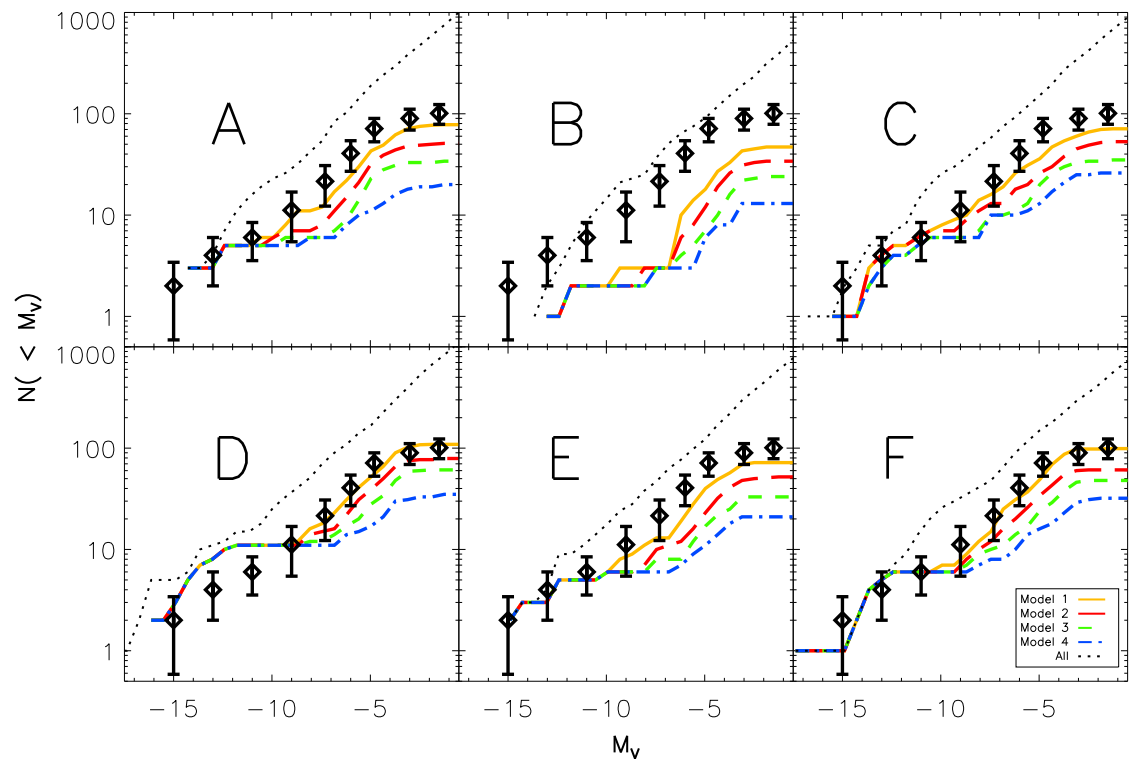

Fig. 11 Observed luminosity function of dwarf galaxies (black diamonds) in comparison with models employing four different physically motivated reionization histories for the six Aquarius halos. Note the scatter in the numbers of simulated satellites among the halos (factor of 2-3) as well as similar variations of the faint end caused by the different reionization histories. The dotted lines depict satellite population unaffected by reionization. From Lunnan et al. (2011).

halos with truly old stars that formed before reionization is of critical importance so it can be established that today's metal-poor stars indeed trace the earliest times and enrichment events. Using the cosmological simulations together with prescriptions for luminous matter, feedback processes and chemical evolution (e.g., Cooper et al. 2010) will shed more light on the cosmological origin of the most metal-poor stars. Stellar and dwarf archaeology meet near-field cosmology for exactly these kinds of questions, and only the combination of high-quality observations and powerful cosmological simulations will enable the progress that is required to understand the early star and galaxy formation and the evolution of our own Milky Way.

\section{Outlook}

Old metal-poor stars have long been used to learn about the conditions of the early Universe. This includes the origin and evolution of the chemical elements, the relevant nucleosynthesis processes and sites and the overall chemical and dynamical history of the Galaxy. By extension, metal-poor stars provide constraints on the na- 
ture of the first stars and their initial mass function, the chemical yields of first/early $\mathrm{SNe}$, as well as early star and galaxy formation processes including the formation of the galactic halo.

Supported by these large-scale survey efforts, the field of near-field cosmology has been catapulted into an "excited state" because it constrains many astrophysical problems related to galaxy formation. It is thus very timely to extensively pursue research into the oldest stars and stellar populations in the dwarf galaxies. They not only enable us to study the history of galaxies with different masses and luminosities, but also facilitate an in-depth study of what role small(er) galaxies may have played in the build-up of the stellar halo.

\subsection{Observational Challenges}

Finding more of the most metal-poor stars (e.g., stars with $[\mathrm{Fe} / \mathrm{H}]<-5.0$ ) is of great importance for addressing the topics described in this chapter in more detail. However, as outlined these stars are extremely rare (Schörck et al. 2009) and difficult to find. The most promising way forward is to survey larger volumes further out in the Galactic halo. But how feasible is it to identify stars with even lower metallicities? Frebel et al. (2009) calculated the minimum observable $\mathrm{Fe}$ and $\mathrm{Mg}$ abundances in the Galaxy by combining the critical metallicity of $[\mathrm{C} / \mathrm{H}]_{\min }=-3.5$ (the criterion for the formation of the first low-mass stars by Bromm \& Loeb (2003) with the maximum carbon-to-iron ratio found in any metal-poor star. The resulting minimum $\mathrm{Fe}$ value is $[\mathrm{Fe} / \mathrm{H}]_{\min }=-7.3$. Analogously, the minimum $\mathrm{Mg}$ value is $[\mathrm{Mg} / \mathrm{H}]_{\min }=-5.5$. If $[\mathrm{C} / \mathrm{H}]_{\min }$ was lower, e.g., $[\mathrm{C} / \mathrm{H}]_{\min }=-4.5$, as suggested by recent dust cooling computations, the minimum observable Fe and $\mathrm{Mg}$ abundances would accordingly be lower. Spectrum synthesis calculations suggest these low abundance levels are indeed measurable from each of the strongest $\mathrm{Fe}$ and $\mathrm{Mg}$ lines in suitably cool metal-deficient giants.

Future surveys such as Skymapper (Keller et al. 2007) and LAMOST will provide an abundance of new metal-poor candidates as well as new, faint dwarf galaxies. By accessing such stars in the outer Galactic halo and dwarf galaxies we will be able to gain a more complete census of the chemical and dynamical history of our own Galaxy. Since the lowest metallicity stars are expected to be in the outer halo (e.g., Carollo et al. 2011), their corresponding kinematic properties may prevent them from accreting too much enriched material from the ISM during their lives so that their surface composition would not be altered (i.e., increased; Frebel et al. 2009). Hence, selecting for the most metal-poor candidates will increasingly rely on our ability to combine chemical abundances with kinematic information. Future missions such as GAIA will provide accurate proper motions for many object that currently have no kinematic information available, including for most of the currently known metal-poor giants.

However, many, if not most, of these future metal-poor candidates will be too faint to be followed up with the high-resolution spectroscopy necessary for detailed 
abundance analyses. This is already an issue for many current candidates leaving the outer halo a so far largely unexplored territory: The limit for high-resolution work is $V \sim 19 \mathrm{mag}$, and one night's observing with 6-10 m telescopes is required for the minimum useful signal-to-noise $(S / N)$ ratio of such a spectrum. With the light-collecting power of the next generation of optical telescopes, such as the Giant Magellan Telescope, the thirty Meter Telescope or the European ELT, and if they are equipped with high-resolution spectrographs, it would be possible to not only reach out into the outer halo in search of the most metal-poor stars, but also provide spectra with very high- $S / N$ ratio of somewhat brighter stars. For example, the socalled r-process enhanced stars which provide crucial empirical constraints on the nature of this nucleosynthesis process require exquisite data quality e.g. for uranium and lead measurements that are currently only possible for the very brightest stars (e.g., Frebel et al. 2007).

It appears that the hunt for the metal-deficient stars in dwarf galaxies may have just begun since these dwarfs host nearly a third of the known low-metallicity stars.. The detailed abundance patterns of the stars in UMa II, ComBer, Leo IV, etc. are strikingly similar to that of the Milky Way stellar halo, thus renewing the support for dwarf galaxies as the building blocks of the halo. Future discoveries of additional faint dwarf galaxies will enable the identification of many more metal-poor stars in new, low-luminosity systems. But also the brighter dSphs have to be revisited for their metal-poor content (Kirby et al. 2009). More stars at the lowest metallicities are clearly desired to better quantify the emerging chemical signatures and to solidify our understanding of the early Galaxy assembly process. Together with advances in the theoretical understanding of early star and galaxy formation and SNe yields, a more complete picture of the evolution of the Milky Way Galaxy and other systems may soon be obtained. Only in this way can the hierarchical merging paradigm for the formation of the Milky Way be put on firm observational ground.

\subsection{Constraining the Theoretical Framework}

An important next step towards a full understanding of the infant universe is to combine the phenomenological approach of collecting new observational data with theoretical knowledge about the formation of the first generations of stars and galaxies. This way, tools can be developed that allow for cosmologically motivated interpretations of the abundance patterns of metal-poor stars, in both the halo and dwarf galaxies. The new field of dwarf archaeology promises a more complete understanding of early enrichment events and the processes that led to galaxy formation at the end of the cosmic dark ages. At the same time, this will have profound implications for the search of the major enrichment mechanisms in the early universe and the physical origin of these stars.

Understanding the Milky Way as a whole is thereby of crucial importance. Most importantly, new results have raised the question of how good the Milky Way of in representing a typical, large spiral galaxy. Our Galaxy is often used as a reference, 
especially when comparing its general properties with those derived from simulations of the formation of large galaxies. But recent works, both observationally and theoretically, have shown that our Galaxy has at least several unusual features. The existence of the long discovered Magellanic Cloud satellites have recently garnered significant attention (Boylan-Kolchin et al. 2010, Liu et al. 2011) in this respect. Observational analyses using Sloan Digital Sky Survey data of many other large spiral galaxies confirmed that galaxies like the Milky Way are very unlikely to have two companions as bright as the Magellanic Clouds. Indeed, less than 5\% of galaxies host two such bright companions, and more than 80 percent host no such satellites at all. Previously, Boylan-Kolchin et al. (2010) have examined this issue using the Millennium simulation of Springel et al. (2005) and finding that the Milky Way was unusual in hosting the Magellanic Clouds.

Furthermore, the evolutionary differences between the Milky and Andromeda need to be to established. The Milky Way did not undergo major mergers with other galaxies since nearly 11 billion years, whereas Andromeda underwent many mergers in a recent past (a few billion years). Only then, can be understood how the different formation histories influence the extent of surviving substructure and differences in properties such as the stellar mass, disk radius, and metal-deficient halo between the sister two galaxies. Assessing the degree of "normality" of the Milky Way will be vital for understanding of whether or not the Magellanic Clouds were only recently accreted by the Milky Way, as proposed by Besla et al. (2007) based on new proper motion measurements.

A major step forward would be a detailed understanding of the missing satellite problem. This is of great interest in cosmology, both observationally and theoretically, but also many associated fields e.g, regarding galaxy formation or dwarf galaxy studies. Is the overproduction of halo substructure at $z=0$ in DM simulations really based on physical processes associated with the gas that lights up dark halos, or is it merely an artifact of past simulations? It has been shown that cosmic variance may play an important role, and without properly quantifying this effect, perhaps no conclusions can be drawn at this point in time. Nevertheless, the fact that the Milky Way may not be an ordinary galaxy may partly explain the missing satellite problem as all simulation results are always compared to the observations associated with the Galaxy.

Progress can be made by carrying out simulations that aim at incorporating nearfield cosmological constraints to address, e.g., the underlying physical causes of the missing satellite problems beyond cosmic variance. To learn about the effects that influence the number of small subhalos over the course of the universe, studies are now being carried out that aim at quantifying the impact of physically motivated, patchy, reionization histories on the faintest halos (e.g., Lunnan et al. 2011). While these studies may partly resolve the missing satellite problem, the overall halo-tohalo differences in the populations is of a similar level, preventing strong, global conclusions. The way forward it to quantify the level and extend of variations of the substructure around Milky Way galaxies. Only then a number of specific details about the origin and evolution of subhalos with different masses equivalent to those of a variety of observed dwarf galaxies, perhaps analogous to massive Magellanic 
Cloud-sized objects, classical dwarf Spheroidal galaxies, and even fainter systems, can be understood.

In summary, the details of the many physical processes that govern the evolution of a luminous halo at $\mathrm{z}=0$ will need to be known to conclusively address the formation of large galaxies within the hierarchical assembly paradigm. They will need to be coupled to simulation results, quantified, and compared to the observations. Hopefully, in the not too distant future, there will be unparalleled opportunities to study the assembly of galaxy halos in close connected to the results of the latest observations of dwarf galaxies, halo stars and stellar streams found in the Milky Way. For example, $[\mathrm{Fe} / \mathrm{H}]$ spreads in dwarf galaxies and well-established abundance trends are important to constrain the chemical evolution in dwarf galaxies. These quantities are crucial for the development of prescriptions for the chemical enrichment throughout the hierarchical merging process. Moreover, they constrain, e.g., metal mixing, turbulence and feedback effects in early star forming halos, as simulated in hydrodynamical "ab-initio" simulations (e.g., Greif et al. 2010).

All these works will soon become possible in great detail as large-scale parallel supercomputers will enable ever more realistic simulations of structure formation as part of the early universe, and on large scales down to $\mathrm{z}=0$. On a similar timescale increasingly detailed observations of stars in the halo and ultra-faint dwarfs will become available (e.g., Skymapper). However, observations alone will likely not be able to uncover the underlying physical processes to conclusively confirm the details of the assembly history of the Milky Way stellar halo and the cosmological origin of the ancient $\sim 13$ Gyr old stars which must have formed long before the Milky Way was fully assembled.

Acknowledgements A.F. gratefully acknowledges a Clay Fellowship which is administered by the Smithsonian Astrophysical Observatory.

\section{References}

1. Aoki, W., Arimoto, N., Sadakane, K., Tolstoy, E., Battaglia, G., Jablonka, P., Shetrone, M., Letarte, B., Irwin, M., Hill, V., Francois, P., Venn, K., Primas, F., Helmi, A., Kaufer, A., Tafelmeyer, M., Szeifert, T., \& Babusiaux, C. 2009, A\&A, 502, 569

2. Aoki, W., Beers, T. C., Sivarani, T., Marsteller, B., Lee, Y. S., Honda, S., Norris, J. E., Ryan, S. G., \& Carollo, D. 2008, ApJ, 678, 1351

3. Aoki, W., Frebel, A., Christlieb, N., Norris, J. E., Beers, T. C., Minezaki, T., Barklem, P. S., Honda, S., Takada-Hidai, M., Asplund, M., Ryan, S. G., Tsangarides, S., Eriksson, K., Steinhauer, A., Deliyannis, C. P., Nomoto, K., Fujimoto, M. Y., Ando, H., Yoshii, Y., \& Kajino, T. 2006, ApJ, 639, 897

4. Beers, T. C. \& Christlieb, N. 2005, ARA\&A, 43, 531

5. Beers, T. C., Preston, G. W., \& Shectman, S. A. 1992, AJ, 103, 1987

6. Bergemann, M. \& Gehren, T. 2008, A\&A, 492, 823

7. Besla, G., Kallivayalil, N., Hernquist, L., Robertson, B., Cox, T. J., van der Marel, R. P., \& Alcock, C. 2007, ApJ, 668, 949

8. Bessell, M. S. \& Norris, J. 1984, ApJ, 285, 622

9. Boylan-Kolchin, M., Besla, G., \& Hernquist, L. 2010, ArXiv:1010.4797 
10. Bromm, V. \& Loeb, A. 2003, Nature, 425,812

11. Bromm, V. \& Yoshida, N. 2011, ArXiv: 1102.4638

12. Bromm, V., Yoshida, N., Hernquist, L., \& McKee, C. F. 2009, Nature, 459, 49

13. Busha, M. T., Alvarez, M. A., Wechsler, R. H., Abel, T., \& Strigari, L. E. 2010, ApJ, 710, 408

14. Carollo, D., Beers, T. C., Lee, Y. S., Chiba, M., Norris, J. E., Wilhelm, R., Sivarani, T., Marsteller, B., Munn, J. A., Bailer-Jones, C. A. L., Fiorentin, P. R., \& York, D. G. 2007, Nature, 450, 1020

15. Cayrel, R., Depagne, E., Spite, M., Hill, V., Spite, F., François, P., Plez, B., Beers, T., Primas, F., Andersen, J., Barbuy, B., Bonifacio, P., Molaro, P., \& Nordström, B. 2004, A\&A, 416, 1117

16. Chiappini, C., Frischknecht, U., Meynet, G., Hirschi, R., Barbuy, B., Pignatari, M., Decressin, T., \& Maeder, A. 2011, Nature, 472, 454

17. Christlieb, N., Beers, T. C., Barklem, P. S., Bessell, M., Hill, V., Holmberg, J., Korn, A. J., Marsteller, B., Mashonkina, L., Qian, Y.-Z., Rossi, S., Wasserburg, G. J., Zickgraf, F.-J., Kratz, K.-L., Nordström, B., Pfeiffer, B., Rhee, J., \& Ryan, S. G. 2004, A\&A, 428, 1027

18. Christlieb, N., Bessell, M. S., Beers, T. C., Gustafsson, B., Korn, A., Barklem, P. S., Karlsson, T., Mizuno-Wiedner, M., \& Rossi, S. 2002, Nature, 419, 904

19. Christlieb, N., Schörck, T., Frebel, A., Beers, T. C., Wisotzki, L., \& Reimers, D. 2008, A\&A, 484, 721

20. Clark, P. C., Glover, S. C. O., Smith, R. J., Greif, T. H., Klessen, R. S., \& Bromm, V. 2011, Science, 331, 1040

21. Cohen, J. G., Christlieb, N., McWilliam, A., Shectman, S., Thompson, I., Melendez, J., Wisotzki, L., \& Reimers, D. 2008, ApJ, 672, 320

22. Cohen, J. G. \& Huang, W. 2009, ApJ, 701, 1053

23. Cooper, A. P., Cole, S., Frenk, C. S., White, S. D. M., Helly, J., Benson, A. J., De Lucia, G., Helmi, A., Jenkins, A., Navarro, J. F., Springel, V., \& Wang, J. 2010, MNRAS, 406, 744

24. Diemand, J., Kuhlen, M., \& Madau, P. 2007, ApJ, 667, 859

25. Feltzing, S., Eriksson, K., Kleyna, J., \& Wilkinson, M. I. 2009, A\&A, 508, L1

26. Font, A. S., Johnston, K. V., Bullock, J. S., \& Robertson, B. E. 2006, ApJ, 646, 886

27. Frebel, A. 2010, Astronomische Nachrichten, 331, 474

28. Frebel, A., Aoki, W., Christlieb, N., Ando, H., Asplund, M., Barklem, P. S., Beers, T. C., Eriksson, K., Fechner, C., Fujimoto, M. Y., Honda, S., Kajino, T., Minezaki, T., Nomoto, K., Norris, J. E., Ryan, S. G., Takada-Hidai, M., Tsangarides, S., \& Yoshii, Y. 2005a, Nature, 434, 871

29. Frebel, A., Aoki, W., Christlieb, N., Ando, H., Asplund, M., Barklem, P. S., Beers, T. C., Eriksson, K., Fechner, C., Fujimoto, M. Y., Honda, S., Kajino, T., Minezaki, T., Nomoto, K., Norris, J. E., Ryan, S. G., Takada-Hidai, M., Tsangarides, S., \& Yoshii, Y. 2005b, in IAU Symposium 228, 207

30. Frebel, A. \& Bromm, V. 2010, ArXiv e-prints 1010.1261

31. Frebel, A., Christlieb, N., Norris, J. E., Beers, T. C., Bessell, M. S., Rhee, J., Fechner, C., Marsteller, B., Rossi, S., Thom, C., Wisotzki, L., \& Reimers, D. 2006, ApJ, 652, 1585

32. Frebel, A., Christlieb, N., Norris, J. E., Thom, C., Beers, T. C., \& Rhee, J. 2007a, ApJ, 660, L117

33. Frebel, A., Johnson, J. L., \& Bromm, V. 2007b, MNRAS, 380, L40

34. - 2009, MNRAS, 392, L50

35. Frebel, A., Kirby, E. N., \& Simon, J. D. 2010a, Nature, 464, 72

36. Frebel, A. \& Norris, J. E. 2011, 1102.1748

37. Frebel, A., Simon, J. D., Geha, M., \& Willman, B. 2010b, ApJ, 708, 560

38. Fulbright, J. P., Rich, R. M., \& Castro, S. 2004, ApJ, 612, 447

39. Geha, M., Willman, B., Simon, J. D., Strigari, L. E., Kirby, E. N., Law, D. R., \& Strader, J. 2009, ApJ, 692, 1464

40. Geisler, D., Smith, V. V., Wallerstein, G., Gonzalez, G., \& Charbonnel, C. 2005, AJ, 129, 1428

41. Greif, T. H., Glover, S. C. O., Bromm, V., \& Klessen, R. S. 2010, ApJ, 716, 510

42. Greif, T. H., Johnson, J. L., Klessen, R. S., \& Bromm, V. 2008, MNRAS, 387, 1021 
43. Heger, A. \& Woosley, S. E. 2002, ApJ, 567, 532

44. -. 2010, ApJ, 724, 341

45. Hollek, J. K., Frebel, A., Roederer, I. U., Sneden, C., Shetrone, M., Beers, T., Kang, S.-J., Thom, C., and Lambert. D. ApJ, submitted

46. Iben, I. 1983, Memorie della Societa Astronomica Italiana, 54, 321

47. Iwamoto, N., Umeda, H., Tominaga, N., Nomoto, K., \& Maeda, K. 2005, Science, 309, 451

48. Johnson, J. L. \& Khochfar, S. 2011, MNRAS, 413, 1184

49. Johnston, K. V., Bullock, J. S., Sharma, S., Font, A., Robertson, B. E., \& Leitner, S. N. 2008, ApJ, 689, 936

50. Karlsson, T. \& Gustafsson, B. 2005, A\&A, 436, 879

51. Keller, S. C., Schmidt, B. P., Bessell, M. S., Conroy, P. G., Francis, P., Granlund, A., Kowald, E., Oates, A. P., Martin-Jones, T., Preston, T., Tisserand, P., Vaccarella, A., \& Waterson, M. F. 2007, Publications of the Astronomical Society of Australia, 24, 1

52. Kirby, E. N., Guhathakurta, P., Bolte, M., Sneden, C., \& Geha, M. C. 2009, ApJ, 705, 328

53. Kirby, E. N., Guhathakurta, P., \& Sneden, C. 2008a, ApJ, 682, 1217

54. Kirby, E. N., Lanfranchi, G. A., Simon, J. D., Cohen, J. G., \& Guhathakurta, P. 2011, ApJ, 727,78

55. Kirby, E. N., Simon, J. D., Geha, M., Guhathakurta, P., \& Frebel, A. 2008b, ApJ, 685, L43

56. Klypin, A., Kravtsov, A. V., Valenzuela, O., \& Prada, F. 1999, ApJ, 522, 82

57. Koch, A. 2009, Astronomische Nachrichten, 330, 675

58. Koch, A., McWilliam, A., Grebel, E. K., Zucker, D. B., \& Belokurov, V. 2008, ApJ, 688, L13

59. Komatsu, E., Smith, K. M., Dunkley, J., Bennett, C. L., Gold, B., Hinshaw, G., Jarosik, N., Larson, D., Nolta, M. R., Page, L., Spergel, D. N., Halpern, M., Hill, R. S., Kogut, A., Limon, M., Meyer, S. S., Odegard, N., Tucker, G. S., Weiland, J. L., Wollack, E., \& Wright, E. L. 2011, ApJS, 192, 18

60. Lai, D. K., Bolte, M., Johnson, J. A., \& Lucatello, S., Heger, A. and Woosley, S. E. 2008, ApJ, 681,1524

61. Li, H. N., Christlieb, N., Schörck, T., Norris, J. E., Bessell, M. S., Yong, D., Beers, T. C., Lee, Y. S., Frebel, A., \& Zhao, G. 2010, A\&A, 521, A10

62. Limongi, M., Chieffi, A., \& Bonifacio, P. 2003, ApJ, 594, L123

63. Liu, L., Gerke, B. F., Wechsler, R. H., Behroozi, P. S., \& Busha, M. T. 2010, ArXiv:1011.2255

64. Lunnan, R., Vogelsberger, M., Frebel, A., Hernquist, L., Lidz, A., \& Boylan-Kolchin, M. 2011 , submitted to ApJ; see www.cfa.harvard.edu/ afrebel/lunnan_etal. ps for a version

65. McQuinn, M., Lidz, A., Zahn, O., Dutta, S., Hernquist, L., \& Zaldarriaga, M. 2007, MNRAS, 377, 1043

66. McWilliam, A., Preston, G. W., Sneden, C., \& Searle, L. 1995, AJ, 109, 2757

67. Meynet, G., Ekström, S., \& Maeder, A. 2006a, A\&A, 447, 623

68. - 2006b, A\&A, 447, 623

69. Moore, B., Ghigna, S., Governato, F., Lake, G., Quinn, T., Stadel, J., \& Tozzi, P. 1999, ApJ, 524, L19

70. Munoz, J. A., Madau, P., Loeb, A., \& Diemand, J. 2009, MNRAS, 400, 1593

71. Norris, J. E., Gilmore, G., Wyse, R. F. G., Wilkinson, M. I., Belokurov, V., Evans, N. W., \& Zucker, D. B. 2008, ApJ, 689, L113

72. Norris, J. E., Gilmore, G., Wyse, R. F. G., Yong, D., \& Frebel, A. 2010a, ApJ, 722, L104

73. Norris, J. E., Ryan, S. G., Beers, T. C., \& Deliyannis, C. P. 1997, ApJ, 485, 370

74. Norris, J. E., Yong, D., Gilmore, G., \& Wyse, R. F. G. 2010b, ApJ, 711, 350

75. Robertson, B., Bullock, J. S., Font, A. S., Johnston, K. V., \& Hernquist, L. 2005, ApJ, 632, 872

76. Ryan, S. G., Norris, J. E., \& Beers, T. C. 1996, ApJ, 471, 254

77. - 1999, ApJ, 523, 654

78. Schneider, R., Omukai, K., Inoue, A. K., \& Ferrara, A. 2006, MNRAS, 369, 1437

79. Schörck, T., Christlieb, N., Cohen, J. G., Beers, T. C., Shectman, S., Thompson, I., McWilliam, A., Bessell, M. S., Norris, J. E., Meléndez, J., Ramírez, S., Haynes, D., Cass, P., Hartley, M., Russell, K., Watson, F., Zickgraf, F., Behnke, B., Fechner, C., Fuhrmeister, B., Barklem, P. S., Edvardsson, B., Frebel, A., Wisotzki, L., \& Reimers, D. 2009, A\&A, 507, 817 
80. Searle, L. \& Zinn, R. 1978, ApJ, 225, 357

81. Shetrone, M., Venn, K. A., Tolstoy, E., Primas, F., Hill, V., \& Kaufer, A. 2003, AJ, 125, 684

82. Shigeyama, T., Tsujimoto, T., \& Yoshii, Y. 2003, ApJ, 586, L57

83. Simmerer, J., Sneden, C., Cowan, J. J., Collier, J., Woolf, V. M., \& Lawler, J. E. 2004, ApJ, 617,1091

84. Simon, J. D., Frebel, A., McWilliam, A., Kirby, E. N., \& Thompson, I. B. 2010, ApJ, 716, 446

85. Simon, J. D. \& Geha, M. 2007, ApJ, 670, 313

86. Sobeck, J. S., Lawler, J. E., \& Sneden, C. 2007, ApJ, 667, 1267

87. Spergel, D. N., Bean, R., Doré, O., Nolta, M. R., Bennett, C. L., Dunkley, J., Hinshaw, G., Jarosik, N., Komatsu, E., Page, L., Peiris, H. V., Verde, L., Halpern, M., Hill, R. S., Kogut, A., Limon, M., Meyer, S. S., Odegard, N., Tucker, G. S., Weiland, J. L., Wollack, E., \& Wright, E. L. 2007, ApJS, 170, 377

88. Springel, V. 2005, MNRAS, 364, 1105

89. Springel, V., Wang, J., Vogelsberger, M., Ludlow, A., Jenkins, A., Helmi, A., Navarro, J. F., Frenk, C. S., \& White, S. D. M. 2008, MNRAS, 391, 1685

90. Springel, V., White, S. D. M., Jenkins, A., Frenk, C. S., Yoshida, N., Gao, L., Navarro, J., Thacker, R., Croton, D., Helly, J., Peacock, J. A., Cole, S., Thomas, P., Couchman, H., Evrard, A., Colberg, J., \& Pearce, F. 2005, Nature, 435, 629

91. Stacy, A., Greif, T. H., \& Bromm, V. 2010, MNRAS, 403, 45

92. Starkenburg, E., Hill, V., Tolstoy, E., González Hernández, J. I., Irwin, M., Helmi, A., Battaglia, G., Jablonka, P., Tafelmeyer, M., Shetrone, M., Venn, K., \& de Boer, T. 2010, A\&A, 513, A34

93. Suda, T., Aikawa, M., Machida, M. N., Fujimoto, M. Y., \& Iben, I. J. 2004, ApJ, 611, 476

94. Tafelmeyer, M., Jablonka, P., Hill, V., Shetrone, M., Tolstoy, E., Irwin, M. J., Battaglia, G., Helmi, A., Starkenburg, E., Venn, K. A., Abel, T., Francois, P., Kaufer, A., North, P., Primas, F., \& Szeifert, T. 2011, A\&A, 527, C1+

95. Tolstoy, E., Hill, V., \& Tosi, M. 2009, ARA\&A, 47, 371

96. Tominaga, N., Umeda, H., \& Nomoto, K. 2007, ApJ, 660, 516

97. Umeda, H. \& Nomoto, K. 2003, Nature, 422, 871

98. Wisotzki, L., Köhler, T., Groote, D., \& Reimers, D. 1996, A\&AS, 115, 227

99. Woosley, S. E. \& Weaver, T. A. 1995, ApJS, 101, 181

100. Yoshida, N., Omukai, K., Hernquist, L., \& Abel, T. 2006, ApJ, 652, 6

101. Zahn, O., Lidz, A., McQuinn, M., Dutta, S., Hernquist, L., Zaldarriaga, M., \& Furlanetto, S. R. 2007, ApJ, 654, 12 